J. DIFFERENTIAL GEOMETRY

86 (2010) 237-271

\title{
EXPLICIT BIRATIONAL GEOMETRY OF 3-FOLDS OF GENERAL TYPE, II
}

\author{
Jungkai A. Chen \& Meng Chen
}

\begin{abstract}
Let $V$ be a complex nonsingular projective 3 -fold of general type. We shall give a detailed classification up to baskets of singularities on a minimal model of $V$. We show that the $m$-canonical map of $V$ is birational for all $m \geq 73$ and that the canonical volume $\operatorname{Vol}(V) \geq \frac{1}{2660}$. When $\chi\left(\mathcal{O}_{V}\right) \leq 1$, our result is $\operatorname{Vol}(V) \geq \frac{1}{420}$, which is optimal. Other effective results are also included in the paper.
\end{abstract}

\section{Introduction}

Let $Y$ be a nonsingular projective variety of dimension $n$. It is said to be of general type if the pluricanonical map $\varphi_{m}$ corresponding to the linear system $\left|m K_{Y}\right|$ is birational into a projective space for $m \gg 0$. Thus it is natural and important to find a constant $c(n)$, depending only on dimension, so that $\varphi_{m}$ is birational onto its image for all $m \geq c(n)$ and for all $Y$ with $\operatorname{dim} Y=n$.

It was classically known that, when $\operatorname{dim} Y=1,\left|m K_{Y}\right|$ gives an embedding of $Y$ into a projective space for $m \geq 3$. When $\operatorname{dim} Y=2$, Kodaira-Bombieri's theorem [2] implies that $\left|m K_{Y}\right|$ gives a birational map onto the image for $m \geq 5$. A recent result of Hacon and $\mathrm{M}^{\mathrm{c}}$ Kernan [10], Takayama [23], and Tsuji [25] shows the existence of $c(n)$, which is however non-explicit.

This is the continuation of our previous paper [4]. The aim of this paper is to prove a practical constant $c(3)$, which is not too far from being sharp. Other effective results are included in this paper as well.

Recall that we have proved the following result in $[4]$.

Theorem 1. ([4, Theorem 1.1]) Let $V$ be a nonsingular projective 3-fold of general type. Then:

(1) $P_{12}>0$

(2) $P_{m_{0}} \geq 2$ for some positive integer $m_{0} \leq 24$.

Our main theorems of this paper are as follows.

Received 1/8/2010. 
Theorem 1.1. Let $V$ be a nonsingular projective 3-fold of general type. Then:

(1) $P_{m}>0$ for all $m \geq 27$.

(2) $P_{24} \geq 2$ and $P_{m_{0}} \geq 2$ for some positive integer $m_{0} \leq 18$.

(3) $\varphi_{m}$ is birational for all $m \geq 73$, and in case $\chi\left(\mathcal{O}_{X}\right) \leq 1, \varphi_{m}$ is birational for all $m \geq 40$.

Here is our result on the volume.

Theorem 1.2. Let $V$ be a non-singular projective 3-fold of general type. Then:

(1) $\operatorname{Vol}(V) \geq \frac{1}{2660}$. Furthermore, $\operatorname{Vol}(V)=\frac{1}{2660}$ if and only if $P_{2}=0$ and either $\chi\left(\mathcal{O}_{V}\right)=3, \mathscr{B}(X)=\left\{9 \times \frac{1}{2}(1,-1,1)\right.$, $2 \times \frac{1}{7}(1,-1,3), \frac{1}{19}(1,-1,7), 3 \times \frac{1}{3}(1,-1,1), \frac{1}{10}(1,-1,3), \frac{1}{4}(1,-1,1)$, $\left.\frac{1}{5}(1,-1,1)\right\}$ or $\chi\left(\mathcal{O}_{V}\right)=2, \mathscr{B}(X)=\left\{2 \times \frac{1}{2}(1,-1,1), 2 \times \frac{1}{7}(1,-1,3)\right.$, $\left.2 \times \frac{1}{5}(1,-1,2), \frac{1}{19}(1,-1,7), \frac{1}{4}(1,-1,1)\right\}$ where $\mathscr{B}(X)$ is the basket of singularities on a minimal model $X$ of $V$.

(2) In case $\chi\left(\mathcal{O}_{V}\right) \leq 1, \operatorname{Vol}(V) \geq \frac{1}{420}$, which is an optimal lower bound. Furthermore, $\operatorname{Vol}(V)=\frac{1}{420}$ if and only if the basket of singularities on any minimal model $X$ of $V$ is

$$
\left\{3 \times \frac{1}{2}(1,-1,1), \frac{1}{7}(1,-1,3), \frac{1}{5}(1,-1,2), \frac{1}{4}(1,-1,1), \frac{1}{6}(1,-1,1)\right\} .
$$

Theorem $1.2(2)$ is optimal due to the following example:

Example 1.3. ([12, page 151, no. 23] ) The canonical hypersurface $X_{46} \subset \mathbb{P}(4,5,6,7,23)$ has 7 terminal quotient singularities and the canonical volume $K_{X_{46}}^{3}=\frac{1}{420}$. One knows $\chi\left(\mathcal{O}_{X_{46}}\right)=1$ since $p_{g}\left(X_{46}\right)=q\left(X_{46}\right)=h^{2}\left(\mathcal{O}_{X_{46}}\right)=0$. Furthermore, it is known that $\varphi_{m}$ is birational for all $m \geq 27$, but $\varphi_{26}$ is not birational.

We now briefly sketch the main idea of this article. A general approach to study pluricanonical maps in higher dimensions is by utilizing vanishing theorems. The difficulty is usually reduced to bound from below the canonical volume

$$
\operatorname{Vol}(Y):=\limsup _{\left\{m \in \mathbb{Z}^{+}\right\}}\left\{\frac{n !}{m^{n}} \operatorname{dim}_{\mathbb{C}} H^{0}\left(Y, \mathcal{O}_{Y}\left(m K_{Y}\right)\right)\right\} .
$$

The volume is an integer when $\operatorname{dim} Y \leq 2$, and hence a naive lower bound 1 is obtained. However, it's a rational number in dimension three or higher. This is an essential difficulty of high-dimensional birational geometry.

Another technical approach is the induction approach initiated by Kollár [15], who proved that $\varphi_{11 m_{0}+5}$ is birational provided $P_{m_{0}} \geq 2$ for 3-folds of general type. Kollár's method has been generalized in several directions by Chen [7], Chen-Hacon [3], Chen-Zuo [8], ChenChen [5], and so on. Therefore, it remains to consider 3 -folds with 
small plurigenera. One notices that the plurigenus $P_{m}(Y)$ is nothing but the Euler characteristic $\chi\left(X, m K_{X}\right)$ of its minimal model $X$ thanks to the vanishing theorem, and moreover if the minimal model is nonsingular or Gorenstein, then $\chi\left(\mathcal{O}_{Y}\right)<0$. One obtains $P_{2} \geq 4$ easily by the Riemann-Roch formula.

Reid introduced the notion of baskets of singularities which are local deformation of singularities into cyclic quotients and derived a singular Riemann-Roch formula for threefolds with at worst canonical singularities. Roughly speaking, the "singular" Riemann-Roch formula computes the Euler characteristic $\chi\left(Y, m K_{Y}\right)$ by the usual Riemann-Roch terms and the contribution from singularities which is computed by baskets. The key new ingredient is our systematical study of baskets of singularities in [4]. Our method provides a concrete way to determine an approximation of a basket with given leading Euler characteristics $\chi\left(K_{Y}\right), \chi\left(2 K_{Y}\right), \cdots$, etc. As a consequence, we are able to prove the finiteness of baskets with small leading Euler characteristics. It is even possible to give explicit classification up to baskets, which is exactly what we have done in this paper.

The article is organized as follows. In Section 2, we summarize some results on the geometry of $|m K|$, which substantially extend the above mentioned technique. Combining with the technique on baskets of singularities developed in [4], we will give a successful classification in case $\chi(\mathcal{O})=1$ in Section 3. In Section 4, we classify baskets such that $P_{m} \leq 1$ for all $1<m \leq 12$ and $\chi(\mathcal{O})>1$. We get 63 classes of baskets of 3-folds in Table C. All these classification allows us to find a practical number $n_{1}>0$ such that $P_{n_{1}} \geq 2$. Therefore, we are able to prove our main theorems.

Throughout, we will frequently use those definitions, equalities, and inequalities about formal baskets in our previous paper (see [4, Sections 3 and 4]). We prefer to use "三" to denote numerical equivalence, while " $\sim$ " represents linear equivalence. Roundup operator " $\lceil *\rceil$ " is defined to be " $-\lfloor-*\rfloor "$, where rounddown " $\lfloor *\rfloor$ " means taking the integral part.

We are very grateful to an anonymous referee whose keen suggestion makes this paper be much better organized.

Acknowledgments. The first author was partially supported by TIMS, NCTS/TPE and National Science Council of Taiwan. The second author was supported by National Outstanding Young Scientist Foundation (\#10625103) and NNSFC Key project (\#10731030).

\section{Technical preparation}

In this section, we set up some notions and principles evolved in our detailed study. We shall prove some general results on pluricanonical birationality and the lower bound of canonical volume. Though the 
method has already appeared in several previous works, the way of applying it is resultful to the effect that we are able to treat various situations while proving our main theorems.

2.1. Reduction to problems on minimal 3-folds. Let $V$ be a non-singular projective 3 -fold of general type. By the 3-dimensional Minimal Model Program (see, for instance, $[\mathbf{1 4}, \mathbf{1 6}, \mathbf{2 0}]$ ), $V$ has a minimal model $X$ (with $K_{X}$ nef and admitting $\mathbb{Q}$-factorial terminal singularities). Denote by $K_{X}$ a canonical divisor of $X$. A basic fact is that $\operatorname{Vol}(V)=K_{X}^{3}>0$. From the view point of birational geometry, it suffices to prove main theorem for minimal 3 -folds $X$.

Definition 2.2. (1) The number $\rho_{i}=\rho_{i}(X)$ denotes the minimal positive integer such that $P_{m}(X)>i$ for all $m \geq \rho_{i}$, where $i=0,1$.

(2) The number $\mu_{i}=\mu_{i}(X)$ denotes the minimal positive integer with $P_{\mu_{i}}=P_{\mu_{i}}(X)>i$ where $i=0,1,2$.

(3) Denote by $\mathscr{B}(X)$ the basket of singularities on $X$ (according to Reid [21]), and by $r(X)$ the Cartier index of $X$.

By our definition, we see $\rho_{0} \leq \rho_{1}$ and $\mu_{0} \leq \mu_{1} \leq \rho_{1}$. The existence of $\rho_{1}$ can be guaranteed by Theorem 1 .

Now suppose we have $P_{m_{0}} \geq 2$ for certain positive integer $m_{0}$. We may study the geometry of the rational map $\varphi_{m_{0}}:=\Phi_{\left|m_{0} K_{X}\right|}$.

2.3. Set up for $\varphi_{m_{0}}$. We study the $m_{0}$-canonical map of $X$ :

$$
\varphi_{m_{0}}: X \rightarrow \mathbb{P}^{P_{m_{0}}-1},
$$

which is a rational map. First of all we fix an effective Weil divisor $K_{m_{0}} \sim m_{0} K_{X}$. By Hironaka's big theorem, we can take successive blow-ups $\pi: X^{\prime} \rightarrow X$ such that:

(i) $X^{\prime}$ is smooth;

(ii) the movable part of $\left|m_{0} K_{X^{\prime}}\right|$ is base point free;

(iii) the support of the union of $\pi^{*}\left(K_{m_{0}}\right)$ and the exceptional divisors is of simple normal crossings.

Set $g_{m_{0}}:=\varphi_{m_{0}} \circ \pi$. Then $g_{m_{0}}$ is a morphism by assumption. Let $X^{\prime} \stackrel{f}{\longrightarrow} \Gamma \stackrel{s}{\longrightarrow} W^{\prime}$ be the Stein factorization of $g_{m_{0}}$ with $W^{\prime}$ the image of $X^{\prime}$ through $g_{m_{0}}$. In summary, we have the following commutative diagram:

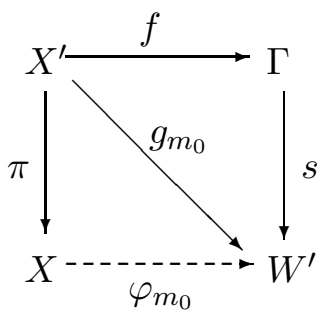


Recall that

$$
\pi^{*}\left(K_{X}\right):=K_{X^{\prime}}-\frac{1}{r(X)} E_{\pi}
$$

with $E_{\pi}$ effective since $X$ is terminal. So we always have

$$
\left\lceil m \pi^{*}\left(K_{X}\right)\right\rceil:=\left\lceil m K_{X^{\prime}}-\frac{m}{r(X)} E_{\pi}\right\rceil \leq m K_{X^{\prime}}
$$

for any integer $m>0$. Denote by $M_{m_{0}}$ the movable part of $\left|m_{0} K_{X^{\prime}}\right|$. One has

$$
m_{0} \pi^{*}\left(K_{X}\right)=M_{m_{0}}+E_{m_{0}}^{\prime}
$$

for an effective $\mathbb{Q}$-divisor $E_{m_{0}}^{\prime}$. In total, since

$$
h^{0}\left(X^{\prime},\left\lfloor m_{0} \pi^{*}\left(K_{X}\right)\right\rfloor\right)=h^{0}\left(X^{\prime},\left\lceil m_{0} \pi^{*}\left(K_{X}\right)\right\rceil\right)=P_{m_{0}}\left(X^{\prime}\right)=P_{m_{0}}(X),
$$

one has

$$
m_{0} K_{X^{\prime}}=M_{m_{0}}+\left(E_{m_{0}}^{\prime}+\frac{m_{0}}{r(X)} E_{\pi}\right)
$$

where $E_{m_{0}}^{\prime}+\frac{m_{0}}{r(X)} E_{\pi}$ is exactly the fixed part of $\left|m_{0} K_{X^{\prime}}\right|$.

If $\operatorname{dim}(\Gamma) \geq 2$, a general member $S$ of $\left|M_{m_{0}}\right|$ is a nonsingular projective surface of general type by Bertini's theorem and by the easy addition formula for Kodaira dimension.

If $\operatorname{dim}(\Gamma)=1$, a general fiber $S$ of $f$ is an irreducible smooth projective surface of general type, still by the easy addition formula for Kodaira dimension. We may write

$$
M_{m_{0}}=\sum_{i=1}^{a_{m_{0}}} S_{i} \equiv a_{m_{0}} S
$$

where $S_{i}$ are smooth fibers of $f$ for all $i$ and $a_{m_{0}} \geq \min \left\{2 P_{m_{0}}-2, P_{m_{0}}+\right.$ $g(\Gamma)-1\}$, by considering the degree of the divisor $f_{*}\left(M_{0}\right)$ on $\Gamma$.

Definition 2.4. We call $S$ (in 2.3) a generic irreducible element of the linear system $\left|M_{m_{0}}\right|$. Denote by $\sigma: S \longrightarrow S_{0}$ the blow-down onto the smooth minimal model $S_{0}$. By abuse of concepts, we define a generic irreducible element of an arbitrary movable linear system on any projective variety in a similar way.

Definition 2.5. (1) Define the positive integer $p=p\left(m_{0}\right)$ as follows:

$$
p= \begin{cases}1 & \text { if } \operatorname{dim}(\Gamma) \geq 2, \\ a_{m_{0}} & \text { if } \operatorname{dim}(\Gamma)=1 .\end{cases}
$$

(2) To simplify our statements, we say that the fibration $f$ is of type III (resp. II, I) if $\operatorname{dim} \Gamma=3$ (resp. 2,1). According to our needs, we 
would like to divide type I into subclasses:

$$
f \text { is of type } \begin{cases}I_{q} & \text { if } g(\Gamma)>0, \\ I_{3} & \text { if } g(\Gamma)=0, P_{m_{0}} \geq 3, \\ I_{p} & \text { if } g(\Gamma)=0, p_{g}(S)>0, \\ I_{n} & \text { if } g(\Gamma)=0, p_{g}(S)=0 .\end{cases}
$$

2.6. Invariants of the fibration. Let $V$ be a smooth projective 3 -fold and $f: V \longrightarrow \Gamma$ a fibration onto a nonsingular curve $\Gamma$. Leray spectral sequence tells that

$$
E_{2}^{p, q}:=H^{p}\left(\Gamma, R^{q} f_{*} \omega_{V}\right) \Longrightarrow E^{n}:=H^{n}\left(V, \omega_{V}\right) .
$$

By Serre duality and [15, Corollary 3.2, Proposition 7.6], one has the torsion-freeness of the sheaves $R^{i} f_{*} \omega_{V}$ and the following formulae:

$$
\begin{aligned}
& h^{2}\left(\mathcal{O}_{V}\right)=h^{1}\left(\Gamma, f_{*} \omega_{V}\right)+h^{0}\left(\Gamma, R^{1} f_{*} \omega_{V}\right), \\
& q(V):=h^{1}\left(\mathcal{O}_{V}\right)=g(\Gamma)+h^{1}\left(\Gamma, R^{1} f_{*} \omega_{V}\right) .
\end{aligned}
$$

2.7. Birationality principles. Let $Y$ be a nonsingular projective variety on which there are two divisors $D$ and $M$. Assume that $|M|$ is base point free. Take the Stein factorization of $\Phi_{|M|}: Y \stackrel{f}{\longrightarrow} W \longrightarrow$ $\mathbb{P}^{0}(Y, M)-1$ where $f$ is a fibration onto a normal variety $W$. Then the rational map $\Phi_{|D+M|}$ is birational onto its image if one of the following conditions is satisfied:

(i) $\left(\left[\mathbf{2 4}\right.\right.$, Lemma 2]) $\operatorname{dim} \Phi_{|M|}(Y) \geq 2,|D| \neq \emptyset$ and $\left.\Phi_{|D+M|}\right|_{S}$ is birational for a general member $S$ of $|M|$.

(ii) $([6, \S 2.1]) \operatorname{dim} \Phi_{|M|}(Y)=1, \Phi_{|D+M|}$ can separate different general fibers of $f$ and $\Phi_{|D+M|} \mid F$ is birational for a general fiber $F$ of $f$.

Remark 2.8. For the condition 2.7(ii), one knows that $\Phi_{|D+M|}$ can separate different general fibers of $f$ whenever $\operatorname{dim} \Phi_{|M|}(Y)=1, W$ is a rational curve and $D$ is an effective divisor. (In fact, since $|M|$ can separate different fibers of $f$, so can $|D+M|$.)

2.9. Assumptions. Let $m$ be a positive integer. Let $|G|$ be a base point free linear system on $S$. Denote by $C$ a generic irreducible element of $|G|$. Assume:

(1) The linear system $\left|m K_{X^{\prime}}\right|$ distinguishes different generic irreducible elements of $\left|M_{m_{0}}\right|$ (namely, $\Phi_{\left|m K_{X^{\prime}}\right|}\left(S^{\prime}\right) \neq \Phi_{\left|m K_{X^{\prime}}\right|}\left(S^{\prime \prime}\right)$ for two different generic irreducible elements $S^{\prime}, S^{\prime \prime}$ of $\left.\left|M_{m_{0}}\right|\right)$.

(2) The linear system $\left|m K_{X^{\prime}}\right|_{\mid S}$ on $S$ (as a sub-linear system of $\left.\left|m K_{X^{\prime} \mid S}\right|\right)$ distinguishes different generic irreducible elements of $|G|$. (Or sufficiently, the complete linear system

$$
\left|K_{S}+\left\lceil(m-1) \pi^{*}\left(K_{X}\right)-S-\frac{1}{p} E_{m_{0}}^{\prime}\right\rceil_{\mid S}\right|
$$


distinguishes different generic irreducible elements of $|G|$.)

2.10. A lower bound of $K^{3}$. We keep the same notation as above. Since $\pi^{*} K_{X}$ is nef and big, there is a rational number $\beta>0$ such that $\left.\pi^{*}\left(K_{X}\right)\right|_{S}-\beta C$ is numerically equivalent to an effective $\mathbb{Q}$-divisor on $S$.

We further define the following quantities:

$$
\begin{aligned}
\xi & :=\left(\pi^{*}\left(K_{X}\right) \cdot C\right)_{X^{\prime}} ; \\
\alpha & :=\left(m-1-\frac{m_{0}}{p}-\frac{1}{\beta}\right) \xi ; \\
\alpha_{0} & :=\lceil\alpha\rceil .
\end{aligned}
$$

One has

$$
K^{3} \geq \frac{p}{m_{0}} \pi^{*}\left(K_{X}\right)^{2} \cdot S \geq \frac{p \beta}{m_{0}}\left(\pi^{*}\left(K_{X}\right) \cdot C\right)=\frac{p \beta}{m_{0}} \xi .
$$

So it is essential to estimate the rational number $\xi:=\left(\pi^{*}\left(K_{X}\right) \cdot C\right)_{X^{\prime}}$ in order to obtain the lower bound of $K^{3}$. We recall the following:

Theorem 2.11. ([8, Theorem 3.1]) Keep the notation as above. The inequality

$$
\xi \geq \frac{\operatorname{deg}\left(K_{C}\right)+\alpha_{0}}{m}
$$

holds if one of the following conditions is satisfied:

(i) $\alpha>1$

(ii) $\alpha>0$ and $C$ is an even divisor, i.e., $C \sim 2 H$ for a divisor $H$ on $S$.

Furthermore, under Assumptions 2.9(1) and 2.9(2), the map $\varphi_{m}:=$ $\Phi_{\left|m K_{X^{\prime}}\right|}$ is birational onto its image if one of the following conditions is satisfied:

(i) $\alpha>2$;

(ii) $\alpha \geq 2$ and $C$ is not a hyper-elliptic curve on $S$.

Remark 2.12. In particular, the inequality $\xi \geq \frac{\operatorname{deg}\left(K_{C}\right)+\alpha_{0}}{m}$ in Theorem 2.11 implies

$$
\xi \geq \frac{\operatorname{deg}\left(K_{C}\right)}{1+\frac{m_{0}}{p}+\frac{1}{\beta}}
$$

since, whenever $m$ is big enough so that $\alpha>1$,

$$
m \xi \geq \operatorname{deg}\left(K_{C}\right)+\alpha_{0} \geq \operatorname{deg}\left(K_{C}\right)+\left(m-1-\frac{m_{0}}{p}-\frac{1}{\beta}\right) \xi .
$$

As long as we have fixed a linear system $|G|$ on $S$, we are able to prove the effective non-vanishing of plurigenera as follows.

Proposition 2.13. Assume $P_{m_{0}} \geq 2$ for some positive integer $m_{0}$. Then $P_{m}(X)>1$ for all integers $m>1+\frac{m_{0}}{p}+\frac{1}{\beta}$. In particular, $\rho_{0} \leq \rho_{1} \leq\left\lfloor 2+\frac{m_{0}}{p}+\frac{1}{\beta}\right\rfloor$. 
Proof. Assume $m>1+\frac{m_{0}}{p}+\frac{1}{\beta}$. Keep the same notation as in 2.3. Put

$$
\mathcal{L}_{m}:=(m-1) \pi^{*}\left(K_{X}\right)-\frac{1}{p} E_{m_{0}}^{\prime} .
$$

Then we have $\left|K_{X^{\prime}}+\left\lceil\mathcal{L}_{m}\right\rceil\right| \subset\left|m K_{X^{\prime}}\right|$. Noting that

$$
\mathcal{L}_{m}-\left.S \equiv\left(m-1-\frac{m_{0}}{p}\right) \pi^{*}\left(K_{X}\right)\right|_{S}
$$

is nef and big, the Kawamata-Viehweg vanishing theorem $([\mathbf{1 3}, \mathbf{2 6}])$ yields the surjective map

$$
H^{0}\left(X^{\prime}, K_{X^{\prime}}+\left\lceil\mathcal{L}_{m}\right\rceil\right) \rightarrow H^{0}\left(S,\left(K_{X^{\prime}}+\left\lceil\mathcal{L}_{m}\right\rceil\right)_{\mid S}\right) .
$$

Since $S$ is a generic irreducible element of a free linear system, one has $\left.\lceil *\rceil\right|_{S} \geq\lceil * \mid S\rceil$ for any divisor $*$ on $X^{\prime}$. It follows that

$$
\left(K_{X^{\prime}}+\left\lceil\mathcal{L}_{m}\right\rceil\right)_{\mid S} \geq K_{X^{\prime} \mid S}+\left\lceil\mathcal{L}_{m \mid S}\right\rceil \sim K_{S}+\left\lceil\left(\mathcal{L}_{m}-S\right)_{\mid S}\right\rceil .
$$

Note that there is an effective $\mathbb{Q}$-divisor $\hat{H}$ on $S$ such that $\left.\frac{1}{\beta} \pi^{*}\left(K_{X}\right)\right|_{S} \equiv$ $C+\hat{H}$. We consider

$$
\mathcal{D}_{m}:=\left(\mathcal{L}_{m}-S\right)_{\mid S}-\hat{H}
$$

on $S$. Then, by assumption, the divisor $\mathcal{D}_{m}-C \equiv\left(m-1-\frac{m_{0}}{p}-\right.$ $\left.\frac{1}{\beta}\right)\left.\pi^{*}\left(K_{X}\right)\right|_{S}$ is nef and big. Thus the Kawamata-Viehweg vanishing theorem again gives the surjective map

$$
H^{0}\left(S, K_{S}+\left\lceil\mathcal{D}_{m}\right\rceil\right) \longrightarrow H^{0}\left(C, K_{C}+D\right),
$$

where $D:=\left.\left\lceil\mathcal{D}_{m}-C\right\rceil\right|_{C}$ is a divisor on $C$. Because $C$ is a generic irreducible element of a free linear system, we have $D \geq\left\lceil\left(\mathcal{D}_{m}-C\right)_{\mid C}\right\rceil$. A simple calculation gives

$$
\operatorname{deg}(D) \geq\left(\mathcal{D}_{m}-C\right) \cdot C=\left(m-1-\frac{m_{0}}{p}-\frac{1}{\beta}\right) \xi=\alpha>0 .
$$

Noting that $g(C) \geq 2$ since $S$ is of general type, Riemann-Roch formula on $C$ gives $h^{0}\left(C, K_{C}+D\right) \geq 2$. Finally, surjective maps (2.3), (2.5) and inequality (2.4) imply the statement.

q.e.d.

We need the following lemma while studying type $I_{p}, I_{n}$, and $I_{3}$ cases.

Lemma 2.14. Let $S$ be a non-singular projective surface of general type. Denote by $\sigma: S \longrightarrow S_{0}$ the blow-down onto its minimal model $S_{0}$. Let $Q$ be a $\mathbb{Q}$-divisor on $S$. Then $h^{0}\left(S, K_{S}+\lceil Q\rceil\right) \geq 2$ under one of the following conditions:

(i) $p_{g}(S)>0, Q \equiv \sigma^{*}\left(K_{S_{0}}\right)+Q_{1}$ for some nef and big $\mathbb{Q}$-divisor $Q_{1}$ on $S$;

(ii) $p_{g}(S)=0, Q \equiv 2 \sigma^{*}\left(K_{S_{0}}\right)+Q_{2}$ for some nef and big $\mathbb{Q}$-divisor $Q_{2}$ on $S$. 
Proof. First of all, $h^{0}\left(S, 2 K_{S}\right)=h^{0}\left(S, 2 K_{S_{0}}\right)>0$ by the RiemannRoch theorem on $S$, which is a surface of general type. Fix an effective divisor $R_{0} \sim l \sigma^{*}\left(K_{S_{0}}\right)$, where $l=1,2$ in cases (i) and (ii), respectively. Then $R_{0}$ is nef and big and $R_{0}$ is 1-connected by [17, Lemma 2.6]. The Kawamata-Viehweg vanishing theorem says $H^{1}\left(S, K_{S}+\lceil Q\rceil-R_{0}\right)=0$, which gives the surjective map

$$
H^{0}\left(S, K_{S}+\lceil Q\rceil\right) \longrightarrow H^{0}\left(R_{0}, K_{R_{0}}+G_{R_{0}}\right)
$$

where $G_{R_{0}}:=\left(\lceil Q\rceil-R_{0}\right)_{\mid R_{0}}$ with $\operatorname{deg}\left(G_{R_{0}}\right) \geq\left(Q-R_{0}\right) R_{0}=Q_{l} \cdot R_{0}>0$. The 1-connectedness of $R_{0}$ allows us to utilize the Riemann-Roch (see [1], Chapter II) as in the usual way. Note that $S$ is of general type. So $K_{S_{0}}^{2}>0$ and $\operatorname{deg}\left(K_{R_{0}}\right)=2 p_{a}\left(R_{0}\right)-2=\left(K_{S}+R_{0}\right) R_{0} \geq 2$. By the Riemann-Roch theorem on the 1-connected curve $R_{0}$, we have

$$
h^{0}\left(R_{0}, K_{R_{0}}+G_{R_{0}}\right) \geq \operatorname{deg}\left(K_{R_{0}}+G_{R_{0}}\right)+1-p_{a}\left(R_{0}\right) \geq p_{a}\left(R_{0}\right) \geq 2 .
$$

Hence $h^{0}\left(S, K_{S}+\lceil Q\rceil\right) \geq 2$.

q.e.d.

Proposition 2.15. Assume $P_{m_{0}} \geq 2$ for some positive integer $m_{0}$. Then $P_{m} \geq 2$ for $m \geq h\left(m_{0}\right)$ under one of the following situations:

(i) $h\left(m_{0}\right)=2 m_{0}+3$ when $f$ is of type $I_{p}$;

(ii) $h\left(m_{0}\right)=3 m_{0}+4$ when $f$ is of type $I_{n}$;

(iii) $h\left(m_{0}\right)=\left\lfloor\frac{3 m_{0}}{2}\right\rfloor+4$ when $f$ is of type $I_{3}$.

In particular, $\rho_{0} \leq \rho_{1} \leq 2 m_{0}+3,3 m_{0}+4,\left\lfloor\frac{3 m_{0}}{2}\right\rfloor+4$, respectively.

Proof. Keep the same notation as in 2.3. When $f$ is of type I, we have $p=a_{m_{0}}$. By [8, Lemma 3.3], there is a sequence of rational numbers $\left\{\hat{\beta}_{n}\right\}$ with $\hat{\beta}_{n} \mapsto \frac{p}{m_{0}+p} \geq \frac{1}{m_{0}+1}$ such that

$$
\pi^{*}\left(K_{X}\right)_{\mid S}-\hat{\beta}_{n} \sigma^{*}\left(K_{S_{0}}\right) \equiv H_{n}
$$

for an effective $\mathbb{Q}$-divisors $H_{n}$.

We consider

$$
\mathcal{D}_{m}^{\prime}:=\left(\mathcal{L}_{m}-S\right)_{\left.\right|_{S}}-\left(m-1-\frac{m_{0}}{p}\right) H_{n} \equiv\left(m-1-\frac{m_{0}}{p}\right) \hat{\beta}_{n} \sigma^{*}\left(K_{S_{0}}\right) .
$$

If, for $m>0, h^{0}\left(S, K_{S}+\left\lceil\mathcal{D}_{m}^{\prime}\right\rceil\right) \geq 2$, then $h^{0}\left(S, K_{S}+\left\lceil\left(\mathcal{L}_{m}-S\right)_{\left.\right|_{S}}\right\rceil\right) \geq$ 2. It follows then that $P_{m} \geq 2$ by surjective map (2.3) and inequality (2.4). We can choose $h\left(m_{0}\right)$ according to the type of $f$.

When $f$ is of type $I_{p}$, we can pick a big number $n$ so that $\hat{\beta}_{n} \geq \frac{1}{m_{0}+1}-$ $\delta$ for some $0<\delta \ll 1$. For $m \geq 2 m_{0}+3$, we see $\left(m-1-\frac{m_{0}}{p}\right) \hat{\beta}_{n}>1$. By Lemma 2.14 and since $p_{g}(S)>0$, we know $h^{0}\left(S, K_{S}+\left\lceil\mathcal{D}_{m}^{\prime}\right\rceil\right) \geq 2$. Thus we may take $h\left(m_{0}\right)=2 m_{0}+3$.

When $f$ is of type $I_{n}$, we still take a big number $n$ so that $\hat{\beta}_{n} \geq \frac{1}{m_{0}+1}-$ $\delta$ for some $0<\delta \ll 1$. But, for $m \geq 3 m_{0}+4$, we have $\left(m-1-\frac{m_{0}}{p}\right) \hat{\beta}_{n}>2$. By Lemma 2.14 again, we see $h^{0}\left(S, K_{S}+\left\lceil\mathcal{D}_{m}^{\prime}\right\rceil\right) \geq 2$. Thus we may take $h\left(m_{0}\right)=3 m_{0}+4$. 
Finally, when $f$ is of type $I_{3}$, we have $p \geq 2$. One may take a big number $n$ so that $\hat{\beta}_{n} \geq \frac{2}{m_{0}+2}-\delta$ for some $0<\delta \ll 1$. For $m \geq\left\lfloor\frac{3 m_{0}}{2}\right\rfloor+4$, we have $\left(m-1-\frac{m_{0}}{p}\right) \hat{\beta}_{n}>2$. Lemma 2.14 implies $h^{0}\left(S, K_{S}+\left\lceil\mathcal{D}_{m}^{\prime}\right\rceil\right) \geq 2$. Thus we may take $h\left(m_{0}\right)=\left\lfloor\frac{3 m_{0}}{2}\right\rfloor+4$. This completes the proof. q.e.d.

Lemma 2.16. Assume $P_{m_{0}}(X) \geq 2$ for some positive integer $m_{0}$. Keep the same notation as in 2.3. Then, for $m \geq \rho_{0}+m_{0}$, Assumptions 2.9 (1) is satisfied if $f$ is of type $I I I, I I, I_{3}, I_{p}$, or $I_{n}$.

Proof. Let $t>0$ be an integer. We consider the linear system $\mid K_{X^{\prime}}+$ $\left\lceil t \pi^{*}\left(K_{X}\right)\right\rceil+M_{m_{0}}|\subset|\left(m_{0}+t+1\right) K_{X^{\prime}} \mid$. Since $K_{X^{\prime}}+\left\lceil t \pi^{*}\left(K_{X}\right)\right\rceil \geq(t+$ 1) $\pi^{*}\left(K_{X}\right)$, we see that $K_{X^{\prime}}+\left\lceil t \pi^{*}\left(K_{X}\right)\right\rceil$ is effective whenever $t+1 \geq \rho_{0}$.

When $f$ is of type $I_{3}, I_{p}$ or $I_{n}$, we necessarily have $g(\Gamma)=0$. Thus, by $\left[\mathbf{2 4}\right.$, Lemma 2] and Remark 2.8, the linear system $\mid K_{X^{\prime}}+\left\lceil t \pi^{*}\left(K_{X}\right)\right\rceil+$ $M_{m_{0}} \mid$ can separate different generic irreducible elements $S$ of $\left|M_{m_{0}}\right|$. q.e.d.

Lemma 2.17. Let $T$ be a non-singular projective surface of general type on which there is a base point free linear system $|G|$. Let $Q$ be an arbitrary $\mathbb{Q}$-divisor on $T$. Then the linear system $\left|K_{T}+\lceil Q\rceil+G\right|$ can distinguish different generic irreducible elements of $|G|$ under one of the following conditions:

(i) $K_{T}+\lceil Q\rceil$ is effective and $|G|$ is not composed with an irrational pencil of curves;

(ii) $Q$ is nef and big and $|G|$ is composed with an irreducible pencil of curves.

Proof. Statement (i) follows from [24, Lemma 2] and Remark 2.8.

For statement (ii), we pick up a generic irreducible element $C$ of $|G|$. Then $G \equiv s C$ where $s \geq 2$ and $C^{2}=0$. Let $C^{\prime}$ be another generic irreducible element. The Kawamata-Viehweg vanishing theorem gives the surjective map

$$
H^{0}\left(T, K_{T}+\lceil Q\rceil+G\right) \longrightarrow H^{0}\left(C, K_{C}+D\right) \oplus H^{0}\left(C^{\prime}, K_{C^{\prime}}+D^{\prime}\right)
$$

where $D:=\left.(\lceil Q\rceil+G-C)\right|_{C}$ and $D^{\prime}:=\left.\left(\lceil Q\rceil+G-C^{\prime}\right)\right|_{C^{\prime}}$ with $\operatorname{deg}(D)>$ $0, \operatorname{deg}\left(D^{\prime}\right)>0$. Since $T$ is of general type, both $C$ and $C^{\prime}$ are curves of genus $\geq 2$. Thus $h^{0}\left(C, K_{C}+D\right)=h^{0}\left(C^{\prime}, K_{C^{\prime}}+D^{\prime}\right)>1$. Thus $\left|K_{T}+\lceil Q\rceil+G\right|$ can distinguish $C$ and $C^{\prime}$ q.e.d.

Lemma 2.18. Assume $P_{m_{0}}(X) \geq 2$ for some positive integer $m_{0}$. Keep the same notation as in 2.3. Take $G:=\left.S\right|_{S}$ for a generic irreducible element $S$ of $\left|M_{m_{0}}\right|$. Then Assumptions 2.9 (2) is satisfied under one of the following situations:

(i) $f$ is of type III and $m \geq \rho_{0}+m_{0}$.

(ii) $f$ is of type $I I$ and $m \geq \max \left\{\rho_{0}+m_{0}, 2 m_{0}+2\right\}$. 
Proof. Since

$$
\begin{aligned}
& K_{S}+\left\lceil(m-1) \pi^{*}\left(K_{X}\right)-S-\frac{1}{p} E_{m_{0}}^{\prime}\right\rceil_{\mid S} \\
\geq & K_{S}+(m-1) \pi^{*}\left(K_{X}\right)_{\mid S}-\left(S+E_{m_{0}}^{\prime}\right)_{\mid S} \\
= & K_{S}+\left(m-m_{0}-1\right) \pi^{*}\left(K_{X}\right)_{\mid S} \\
\geq & \left(m-m_{0}\right) \pi^{*}\left(K_{X}\right)_{\mid S}+G
\end{aligned}
$$

and

$$
\begin{aligned}
& K_{S}+\left(m-m_{0}-1\right) \pi^{*}\left(K_{X}\right)_{\mid S} \\
\geq & K_{S}+\left(m-2 m_{0}-1\right) \pi^{*}\left(K_{X}\right)_{\mid S}+G,
\end{aligned}
$$

Lemma 2.17 implies that $\left|K_{S}+\left\lceil(m-1) \pi^{*}\left(K_{X}\right)-S-\frac{1}{p} E_{m_{0}}^{\prime}\right\rceil_{\mid S}\right|$ can distinguish different generic irreducible elements of $|G|$ respectively. Note that, if $f$ is of type $I I I,|G|$ is not composed with a pencil of curves. We are done.

q.e.d.

Under the condition $P_{m_{0}} \geq 2$, we study the pluricanonical map $\varphi_{m}$ according to the type of $f$.

2.19. Type $I I I$.

When $f$ is of type $I I I$, we have $p=1$ by definition. In this case, $S \sim M_{m_{0}}$ and $|S|$ gives a generically finite morphism. We take $G:=\left.S\right|_{S}$. Then $|G|$ is base point free and $\varphi_{|G|}$ gives a generically finite map. So a generic irreducible element $C \sim G$ is a smooth curve.

If $\varphi_{|G|}$ gives a birational map, then $\operatorname{dim} \varphi_{|G|}(C)=1$ for a general member $C$. The Riemann-Roch and Clifford's theorem on $C$ says $C^{2}=$ $G \cdot C \geq 2$. If $\varphi_{|G|}$ gives a generically finite map of degree $\geq 2$, since $h^{0}(S, G) \geq h^{0}\left(X^{\prime}, S\right)-1 \geq 3$, one gets $C^{2} \geq 2\left(h^{0}(S, G)-2\right) \geq 2$. Either way, we have $C^{2} \geq 2$. So $\operatorname{deg}\left(K_{C}\right)=\left(K_{S}+C\right) \cdot C>2 C^{2} \geq 4$. We see $\operatorname{deg}\left(K_{C}\right) \geq 6$ since it is a even number.

One may take $\beta=\frac{1}{m_{0}}$ since $\left.m_{0} \pi^{*}\left(K_{X}\right)\right|_{S} \geq C$.

Now inequality $(2.2)$ gives $\xi \geq \frac{6}{2 m_{0}+1}$. Take $m=3 m_{0}+2$. Then $\alpha=\left(m-2 m_{0}-1\right) \xi>3$. So, by Theorem $2.11, \xi \geq \frac{10}{3 m_{0}+2}$. It follows from inequality $(2.1)$ that $K^{3} \geq \frac{10}{\left(3 m_{0}+2\right) m_{0}^{2}}$.

We now consider the non-vanishing of plurigenera. By Proposition 2.13 , we have $P_{m} \geq 2$ for all $m>2 m_{0}+1$. Now, if $m=2 m_{0}+$ 1 , the surjective map (2.3) and inequality (2.4) lead us to compute $h^{0}\left(S, K_{S}+\left\lceil\left. m_{0} \pi^{*} K_{X}\right|_{S}\right\rceil\right)$. Let $L$ be a generic irreducible element in $|S|_{S} \mid$. Then $L$ is effective and nef. Since $h^{2}\left(K_{S}+L\right)=0$, one has $h^{0}\left(S, K_{S}+L\right) \geq \chi\left(S, K_{S}+L\right)=\frac{1}{2}\left(K_{S} \cdot L+L^{2}\right)+\chi\left(\mathcal{O}_{S}\right) \geq 2$ by RiemannRoch theorem. Hence $P_{2 m_{0}+1} \geq 2$. Also, $P_{2 m_{0}} \geq P_{m_{0}} \geq 2$. Therefore, we have $P_{m}>1$ for all $m \geq 2 m_{0}$. In particular, $\rho_{0} \leq \rho_{1} \leq 2 m_{0}$.

By Lemmas 2.16 and 2.18, Assumptions 2.9(1) and 2.9(2) are satisfied if $m \geq 3 m_{0}$. Now $\alpha=\left(m-2 m_{0}-1\right) \xi \geq\left(m-2 m_{0}-1\right) \frac{10}{3 m_{0}+2}$. One sees 
that $\alpha>2$ if $m>\frac{13 m_{0}+7}{5}$. Hence $\varphi_{m}$ is birational if

$$
m>\max \left\{3 m_{0}-1, \frac{13 m_{0}+7}{5}\right\} .
$$

We conclude the following:

Theorem 2.20. Assume $P_{m_{0}}(X) \geq 2$ for some positive integer $m_{0}$. If the induced map $f$ is of type III. Then:

1) $\rho_{0} \leq \rho_{1} \leq 2 m_{0}$.

2) $K^{3} \geq \frac{10}{\left(3 m_{0}+2\right) m_{0}^{2}}$.

3) $\varphi_{m}$ is birational if $m>\max \left\{3 m_{0}-1, \frac{13 m_{0}+7}{5}\right\}$.

2.21. Type $I I$.

When $f$ is of type $I I$, we see that $S \sim M_{m_{0}}$. Take $|G|:=|S|_{S} \mid$, which is, clearly, composed with a pencil of curves.

Since a generic irreducible element $C$ of $|G|$ is a smooth curve of genus $\geq 2$, we have $\operatorname{deg}\left(K_{C}\right) \geq 2$. Furthermore, we have $h^{0}(S, G) \geq$ $h^{0}\left(X^{\prime}, S\right)-1 \geq 2$. So $G \equiv \widetilde{a} C$ where $\widetilde{a} \geq h^{0}(S, G)-1 \geq 1$. This means that $\left.m_{0} \pi^{*}\left(K_{X}\right)\right|_{S} \geq\left. S\right|_{S} \geq_{\text {num }} C$. So we may take $\beta=\frac{1}{m_{0}}$.

Now inequality $(2.2)$ gives $\xi \geq \frac{2}{2 m_{0}+1}$. Take $m=3 m_{0}+2$. Then $\alpha>1$. One gets $\xi \geq \frac{4}{3 m_{0}+2}$ by Theorem 2.11. So inequality (2.1) implies $K^{3} \geq \frac{4}{\left(3 m_{0}+2\right) m_{0}^{2}}$.

Exactly the same proof as in Type $I I I$ shows that $\rho_{0} \leq \rho_{1} \leq 2 m_{0}$.

By Lemmas 2.16 and 2.18, Assumptions 2.9(1) and 2.9(2) are satisfied if $m \geq 3 m_{0}$. Now $\alpha=\left(m-2 m_{0}-1\right) \xi \geq\left(m-2 m_{0}-1\right) \frac{4}{3 m_{0}+2}$. One sees that $\alpha>2$ if $m>\frac{7 m_{0}+4}{2}$. Since $\frac{7 m_{0}+4}{2}>3 m_{0}, \varphi_{m}$ is birational if $m>\frac{7 m_{0}+4}{2}$.

We conclude the following:

Theorem 2.22. Assume $P_{m_{0}}(X) \geq 2$ for some positive integer $m_{0}$. If the induced map $f$ is of type II, then:

1) $\rho_{0} \leq \rho_{1} \leq 2 m_{0}$.

2) $K^{3} \geq \frac{4}{\left(3 m_{0}+2\right) m_{0}^{2}}$.

3) $\varphi_{m}$ is birational if $m>\frac{7 m_{0}+4}{2}$.

2.23. Type $I_{q}$.

Since $g(\Gamma)>0$, one sees $q(X)>0$ and hence $X$ is irregular. This case is particularly well-behaved. It's known that $\varphi_{m}$ is birational for all $m \geq 7$ (see $[\mathbf{3}]$ ). Also $K_{X}^{3} \geq \frac{1}{22}$ (see [5]).

\subsection{Type $I_{p}$.}

We have an induced fibration $f: X^{\prime} \longrightarrow \Gamma$ with $g(\Gamma)=0$. By definition, $p=a_{m_{0}} \geq 1$. By assumption, $p_{g}(S)>0$ for a general fiber $S$ of $f$. We take $G:=2 \sigma^{*}\left(K_{S_{0}}\right)$. Then one knows that $|G|$ is base point free (see [9, Theorem 3.1]). Thus $|G|$ is not composed with a pencil and 
a generic irreducible element $C$ is smooth. By [8, Lemma 3.3], we can find a sequence of rational numbers $\left\{\beta_{n}\right\}$ with $\beta_{n} \mapsto \frac{p}{m_{0}+p}$ such that $\pi^{*}\left(K_{X}\right)_{\mid S}-\frac{\beta_{n}}{2} C \equiv H_{n}$ for effective $\mathbb{Q}$-divisors $H_{n}$. We may assume that $\beta \geq \frac{1}{2\left(m_{0}+1\right)}-\delta$ for some $0<\delta \ll 1$.

Since $C \sim 2 \sigma^{*}\left(K_{S_{0}}\right)$,

$$
\operatorname{deg}\left(K_{C}\right)=\left(K_{S}+C\right) \cdot C \geq\left(\left.\pi^{*}\left(K_{X}\right)\right|_{S}+C\right) \cdot C>C^{2} \geq 4 .
$$

Since $\operatorname{deg}\left(K_{C}\right)$ is even, we see $\operatorname{deg}\left(K_{C}\right) \geq 6$.

Now inequality $(2.2)$ gives $\xi \geq \frac{6}{3 m_{0}+3}$. Take $m=4 m_{0}+5$. Then $\alpha=\left(m-1-m_{0}-\frac{1}{\beta}\right) \xi>2$ and Theorem 2.11 gives $\xi \geq \frac{9}{4 m_{0}+5}$. So, by inequality (2.1), one gets $K^{3} \geq \frac{9}{2 m_{0}\left(m_{0}+1\right)\left(4 m_{0}+5\right)}$.

Note that

$$
\begin{aligned}
& K_{S}+\left\lceil(m-1) \pi^{*}\left(K_{X}\right)-S-\frac{1}{p} E_{m_{0}}^{\prime}\right\rceil_{\mid S} \\
\geq & K_{S}+\left\lceil\left(m-m_{0}-1\right) \pi^{*}\left(K_{X}\right)_{\mid S}\right\rceil \\
\geq & K_{S}+\left\lceil\left(m-m_{0}-1\right) \pi^{*}\left(K_{X}\right)_{\mid S}-\frac{3}{\beta_{n}} H_{n}\right\rceil \\
= & K_{S}+\left\lceil Q_{1}\right\rceil+\sigma^{*}\left(K_{S_{0}}\right)+C
\end{aligned}
$$

where $Q_{1}:=\left(m-m_{0}-1\right) \pi^{*}\left(K_{X}\right)_{\mid S}-C-\sigma^{*}\left(K_{S_{0}}\right)-\frac{3}{\beta_{n}} H_{n} \equiv\left(m-m_{0}-\right.$ $\left.1-\frac{3}{\beta_{n}}\right)\left.\pi^{*}\left(K_{X}\right)\right|_{S}$ is nef and big whenever $m \geq 4 m_{0}+5$. By Lemma 2.14(i), $K_{S}+\left\lceil Q_{1}\right\rceil+\sigma^{*}\left(K_{S_{0}}\right)$ is effective. Thus, according to [24, Lemma 2], Assumption $2.9(2)$ is satisfied for $m \geq 4 m_{0}+5$. Since Proposition 2.15 (ii) implies $\rho_{0} \leq 2 m_{0}+3$, Lemma 2.16 (ii) tells that Assumption $2.9(1)$ is satisfied as long as $m \geq 3 m_{0}+3$. Take $m \geq 4 m_{0}+5$. Then $\alpha \geq\left(m-3 m_{0}-3\right) \xi \geq \frac{2 m_{0}+4}{m_{0}+1}>2$. So Theorem 2.11 implies that $\varphi_{m}$ is birational for all $m \geq 4 m_{0}+5$.

We thus summarize:

Theorem 2.25. Assume $P_{m_{0}}(X) \geq 2$ for some positive integer $m_{0}$. If the induced map $f$ is of type $I_{p}$, then:

1) $\rho_{0} \leq \rho_{1} \leq 2 m_{0}+3$.

2) $K^{3} \geq \frac{9}{2 m_{0}\left(m_{0}+1\right)\left(4 m_{0}+5\right)}$.

3) $\varphi_{m}$ is birational if $m \geq 4 m_{0}+5$.

2.26. Type $I_{n}$.

Similar to the type $I_{p}$ case, we have $p \geq 1$. We take $|G|:=\left|4 \sigma^{*}\left(K_{S_{0}}\right)\right|$ which is base point free by a well-known result in $[2]$. Thus $|G|$ is not composed with a pencil and a generic irreducible element $C$ is smooth. Similarly, we can find a sequence of rational numbers $\left\{\beta_{n}\right\}$ with $\beta_{n} \mapsto$ $\frac{p}{m_{0}+p}$ such that $\pi^{*}\left(K_{X}\right)_{\mid S}-\frac{\beta_{n}}{4} C \equiv H_{n}$ for effective $\mathbb{Q}$-divisors $H_{n}$. We may assume that $\beta \geq \frac{1}{4\left(m_{0}+1\right)}-\delta$ for some $0<\delta \ll 1$.

Since $\operatorname{deg}\left(K_{C}\right)>16 \sigma^{*}\left(K_{S_{0}}\right)^{2} \geq 16$ and $\operatorname{deg}\left(K_{C}\right)$ is even, inequality (2.2) gives $\xi \geq \frac{18}{5 m_{0}+5}$. Take $m=6 m_{0}+6$. Then $\alpha=\left(m-1-m_{0}-\frac{1}{\beta}\right) \xi=$ 
$\frac{18}{5}>3$ and Theorem 2.11 gives $\xi \geq \frac{11}{3 m_{0}+3}$. So, by inequality (2.1), one gets $K^{3} \geq \frac{11}{12 m_{0}\left(m_{0}+1\right)^{2}}$.

By Proposition 2.15, we have $P_{m} \geq 2$ for all $m \geq 3 m_{0}+4$. Thus we have the following:

Theorem 2.27. Assume $P_{m_{0}}(X) \geq 2$ for some positive integer $m_{0}$. If the induced map $f$ is of type $I_{n}$, then:

1) $\rho_{0} \leq \rho_{1} \leq 3 m_{0}+4$.

2) $K^{3} \geq \frac{11}{12 m_{0}\left(m_{0}+1\right)^{2}}$.

3) $\varphi_{m}$ is birational if $m \geq 5 m_{0}+6$ (cf. [7, Theorem 0.1]).

\subsection{Type $I_{3}$.}

We take $G_{1}=4 \sigma^{*}\left(K_{S_{0}}\right)$ so as to estimate $K_{X}^{3}$. Then, as seen in $2.26, \operatorname{deg}\left(K_{C}\right) \geq 18$. Being in a better situation with $p=a_{m_{0}}-1 \geq$ 2 , a better number $\beta$ can be found. In fact, by [8, Lemma 3.3], one may take a number sequence $\left\{\beta_{n}\right\}$ with $\beta_{n} \mapsto \frac{p}{4\left(m_{0}+p\right)} \geq \frac{1}{2\left(m_{0}+2\right)}$ such that $\left.\pi^{*}\left(K_{X}\right)\right|_{S}-\beta_{n} C$ is numerically equivalent to an effective $\mathbb{Q}$-divisor. Namely, one may take a number $\beta \geq \frac{1}{2\left(m_{0}+2\right)}-\delta$ for some $0<\delta \ll 1$. Now inequality (2.2) gives $\xi \geq \frac{18}{1+\frac{m_{0}}{2}+\frac{1}{\beta}}$, i.e., $\xi \geq \frac{36}{5\left(m_{0}+2\right)}$ by taking the limit. Hence inequality $(2.1)$ implies $K^{3} \geq \frac{36}{5 m_{0}\left(m_{0}+2\right)^{2}}$.

We take a different $|G|$ on $S$ to study the birationality. In fact, we will take $|G|$ to be the movable part of $\left|2 \sigma^{*}\left(K_{S_{0}}\right)\right|$. A different point from previous ones is that $|G|$ is not always base point free. But since we have the induced fibration $f: X^{\prime} \longrightarrow \Gamma$, we can consider the relative bi-canonical map of $f$, namely, the rational map $\Psi: X^{\prime} \rightarrow \mathbf{P}$ over $\Gamma$. First we can blow up the indeterminacy of $\Psi$ on $X^{\prime}$. Then we can assume, in the birational equivalence sense, that $\Psi$ is a morphism over $B$. By further modifying $\pi$, we can even finally assume that $\pi$ dominates $\Psi$. With this assumption (or by taking a sufficiently good $\pi$ ), we see that $|G|$ is base point free since $|G|$ gives the bicanonical morphism for each general fiber $S$ of $f$.

By Proposition 2.15 and Lemma 2.16, Assumption 2.9(1) is satisfied for $m \geq\left\lfloor\frac{5 m_{0}}{2}\right\rfloor+4$. Recall that we have $p=a_{m_{0}} \geq 2$.

Claim A. Assumption 2.9(2) is satisfied for $m \geq \min \left\{3 m_{0}+6, \rho_{0}+\right.$ $\left.2 m_{0}+2\right\}$.

In fact, the argument of 2.24 works here. A different place is that we have a better bound for $\beta_{n}$ since $p \geq 2$, but we only have $\operatorname{deg}\left(K_{C}\right) \geq 2$. By $\left[\mathbf{8}\right.$, Lemma 3.3], we can find a sequence of rational numbers $\left\{\beta_{n}\right\}$ with $\beta_{n} \mapsto \frac{p}{2\left(m_{0}+p\right)}$ such that $\pi^{*}\left(K_{X}\right)_{\mid S}-\beta_{n}\left(2 \sigma^{*}\left(K_{S_{0}}\right)\right) \equiv H_{n}$ for effective $\mathbb{Q}$-divisors $H_{n}$. We may assume that $\beta \geq \frac{1}{m_{0}+2}-\delta$ for some $0<\delta \ll 1$. 
Now the last three terms of inequality (2.6) can be replaced by

$$
\begin{aligned}
& K_{S}+\left\lceil\left(m-m_{0}-1\right) \pi^{*}\left(K_{X}\right)_{\mid S}\right\rceil \\
\geq & K_{S}+\left\lceil\left(m-m_{0}-1\right) \pi^{*}\left(K_{X}\right)_{\mid S}-\frac{2}{\beta_{n}} H_{n}\right\rceil \\
= & K_{S}+\left\lceil Q_{2}\right\rceil+4 \sigma^{*}\left(K_{S_{0}}\right)
\end{aligned}
$$

where $Q_{2}:=\left(m-m_{0}-1\right) \pi^{*}\left(K_{X}\right)_{\mid S}-4 \sigma^{*}\left(K_{S_{0}}\right)-\frac{2}{\beta_{n}} H_{n} \equiv\left(m-m_{0}-\right.$ $\left.1-\frac{2}{\beta_{n}}\right)\left.\pi^{*}\left(K_{X}\right)\right|_{S}$ is nef and big whenever $m \geq 3 m_{0}+6$. According to a theorem of Xiao [28], $|G|$ is either not composed with a pencil or composed with a rational pencil. Thus, according to $[\mathbf{2 4}$, Lemma 2] and Remark 2.8, Assumption 2.9(2) is satisfied for $m \geq 3 m_{0}+6$. On the other hand, we have an inclusion, $\mathcal{O}_{\Gamma}(2) \hookrightarrow f_{*} \omega_{X^{\prime}}^{m_{0}}$, which naturally gives rise to the inclusion $f_{*} \omega_{X^{\prime} / \Gamma}^{2} \hookrightarrow f_{*} \omega_{X^{\prime}}^{2 m_{0}+2}$. Now Viehweg's semi-positivity theorem [27] implies that $f_{*} \omega_{X^{\prime} / \Gamma}^{2}$ is generated by global sections. Thus $\left|\left(2 m_{0}+2\right) K_{X^{\prime}}\right|_{\mid S}$ can distinguish different generic irreducible elements of $|G|$. So Assumption 2.9(2) is naturally satisfied for all $m \geq \rho_{0}+2 m_{0}+2$. We have proved Claim A.

Finally, we consider the value of $\alpha$. Recall that we may take $\beta \mapsto$ $\frac{p}{2 m_{0}+2 p} \geq \frac{1}{m_{0}+2}$. Inequality $(2.2)$ gives $\xi \geq \frac{2}{1+\frac{m_{0}}{2}+m_{0}+2}=\frac{4}{3\left(m_{0}+2\right)}$. If we take $m=3 m_{0}+4$. Then $\alpha>1$. Theorem 2.11 says $\xi \geq \frac{4}{3 m_{0}+4}$. Eventually, take $m \geq 3 m_{0}+6$. Then $\alpha>2$. Theorem 2.11 implies that $\varphi_{m}$ is birational for all $m \geq 3 m_{0}+6$.

We thus conclude the following:

Theorem 2.29. Assume $P_{m_{0}}(X) \geq 3$ for some positive integer $m_{0}$. If the induced map $f$ is of type $I_{3}$, then:

1) $\rho_{0} \leq \rho_{1} \leq\left\lfloor\frac{3 m_{0}}{2}\right\rfloor+4$.

2) $K^{3} \geq \frac{36}{5 m_{0}\left(m_{0}+2\right)^{2}}$.

3) $\varphi_{m}$ is birational if $m \geq 3 m_{0}+6$.

By collecting all above results, we have the following:

Corollary 2.30. Assume $P_{m_{0}}(X) \geq 2$ for some positive integer $m_{0}$. Then $K^{3} \geq \frac{11}{12 m_{0}\left(m_{0}+1\right)^{2}}$.

\subsection{Volume optimization.}

Indeed, when $m_{0}$ is small, the estimation of $K_{X}^{3}$ could be optimized by recursively applying Theorem 2.11 with a suitable $m$.

For example, suppose $m_{0}=11$ and $f$ is of type $I I I$. Then inequality (2.2) gives $\xi \geq \frac{6}{23}$. Take $m=27$. By Theorem 2.11, we get $\xi \geq \frac{8}{27}$. So inequality (2.1) gives $K^{3} \geq \frac{8}{3267}>\frac{10}{m_{0}^{2}\left(3 m_{0}+2\right)}$.

Let's consider another example with $m_{0}=8$ and $f$ being of type $I I$. Then we may take $\beta=\frac{1}{8}$. Inequality (2.2) gives $\xi \geq \frac{2}{17}$. Take $m=26$. Then $\alpha \geq \frac{18}{17}>1$. Theorem 2.11 gives $\xi \geq \frac{2}{13}$. Take $m=24$. Then 
$\alpha>1$. Again, one gets $\xi \geq \frac{1}{6}$. So inequality (2.1) implies $K^{3} \geq \frac{1}{384}>$ $\frac{4}{m_{0}^{2}\left(3 m_{0}+2\right)}$.

With the idea mentioned above, a patient reader should have no difficulty to check the following table on the lower bound of $K^{3}$ for small $m_{0}$.

\begin{tabular}{ccccccc}
\multicolumn{7}{c}{ Table A } \\
\hline$m_{0}$ & 2 & 3 & 4 & 5 & 6 & 7 \\
\hline$I I I$ & $1 / 3$ & $8 / 81$ & $1 / 22$ & $8 / 325$ & $1 / 72$ & $4 / 441$ \\
$I I$ & $1 / 8$ & $2 / 45$ & $1 / 52$ & $1 / 100$ & $1 / 162$ & $4 / 1029$ \\
$P_{m_{0}} \geq 3$ & $1 / 8$ & $2 / 45$ & $1 / 52$ & $1 / 100$ & $1 / 162$ & $4 / 1029$ \\
$P_{m_{0}} \geq 2$ & $5 / 96$ & $5 / 264$ & $1 / 108$ & $1 / 192$ & $5 / 1554$ & $5 / 2408$ \\
\hline$m_{0}$ & 8 & 9 & 10 & 11 & 12 & \\
\hline$I I I$ & $1 / 160$ & $4 / 891$ & $2 / 625$ & $8 / 3267$ & $1 / 522$ & \\
$I I$ & $1 / 384$ & $2 / 1053$ & $1 / 725$ & $1 / 968$ & $1 / 1224$ & \\
$P_{m_{0}} \geq 3$ & $1 / 384$ & $2 / 1053$ & $1 / 725$ & $1 / 968$ & $1 / 1224$ & \\
$P_{m_{0}} \geq 2$ & $5 / 3456$ & $1 / 954$ & $1 / 1276$ & $5 / 8448$ & $5 / 10764$ & \\
\hline
\end{tabular}

Lemma 2.32. If $f$ is of type $I_{n}$ and $q(X)=0$, then $\chi\left(\mathcal{O}_{X}\right) \leq 1$.

Proof. We have an induced fibration $f: X^{\prime} \longrightarrow \Gamma$ onto the rational curve $\Gamma$. A general fiber $S$ of $f$ is a non-singular projective surface of general type with $p_{g}(S)=0$. Because $\chi\left(\mathcal{O}_{S}\right)>0$, we see $q(S)=0$. This means $f_{*} \omega_{X^{\prime}}=0$ and $R^{1} f_{*} \omega_{X^{\prime}}=0$ since they are both torsion free by [15]. Thus we get by 2.6 the following formulae:

$$
\begin{gathered}
h^{2}\left(\mathcal{O}_{X}\right)=h^{2}\left(\mathcal{O}_{X^{\prime}}\right)=h^{1}\left(f_{*} \omega_{X^{\prime}}\right)+h^{0}\left(R^{1} f_{*} \omega_{X^{\prime}}\right)=0 ; \\
q(X)=q\left(X^{\prime}\right)=g(\Gamma)+h^{1}\left(R^{1} f_{*} \omega_{X^{\prime}}\right)=0 .
\end{gathered}
$$

So we see $\chi\left(\mathcal{O}_{X}\right)=1-q(X)+h^{2}\left(\mathcal{O}_{X}\right)-p_{g}(X) \leq 1$.

q.e.d.

2.33. Miyaoka-Reid inequality on $\mathscr{B}(X)$. We refer to $[4$, Section 2] for the definition of baskets. Assume that Reid's basket of singularities on $X$ is $B_{X}:=\mathscr{B}(X)=\left\{\left(b_{i}, r_{i}\right)\right\}$. According to $[\mathbf{2 1}, 10.3]$, one has

$$
\frac{1}{12} K_{X} \cdot c_{2}(X)=-2 \chi\left(\mathcal{O}_{X}\right)+\sum_{i} \frac{r_{i}^{2}-1}{12 r_{i}}
$$

where $c_{2}(X)$ is defined via the intersection theory by taking a resolution of singularities of $X$. On the other hand, [18, Corollary 6.7] says $K_{X}$. $c_{2}(X) \geq 0$. Thus one has the following inequality:

$$
\sum_{i} r_{i}-24 \chi\left(\mathcal{O}_{X}\right) \geq \sum_{i} \frac{1}{r_{i}}
$$

A direct application of inequality (2.7) is the following:

Corollary 2.34. Suppose that we have a packing between formal baskets $\mathbf{B}:=\left(B, \chi\left(\mathcal{O}_{X}\right), \tilde{P}_{2}\right) \succcurlyeq \mathbf{B}^{\prime}:=\left(B^{\prime}, \chi\left(\mathcal{O}_{X}\right), \tilde{P}_{2}\right)$ and that inequality (2.7) fails for $\mathbf{B}^{\prime}$. Then (2.7) fails for $\mathbf{B}$. 


\section{General type 3-folds with $\chi=1$}

In this section, we always assume $\chi\left(\mathcal{O}_{X}\right)=1$. If there is a small number $m_{0}$ such that $P_{m_{0}}>1$, then one can detect the birational geometry of $X$ by studying $\varphi_{m_{0}}$. Thus a natural question is what practical number $m_{0}$ can be found such that $P_{m_{0}}>1$. This is exactly the motivation of this section. Equivalently, we shall give a complete classification of baskets to those $X$ with $P_{m} \leq 1$ for $m \leq 6$.

3.1. Assumption: $P_{m}(X) \leq 1$ for $1 \leq m \leq 6$.

In fact, $P_{m}$ satisfies the following geometric condition.

Lemma 3.2. Assume $\chi\left(\mathcal{O}_{X}\right)=1$. Then $P_{m+2} \geq P_{m}+P_{2}$ for all $m \geq 2$.

Proof. By Reid's formula ([21]), we have

$$
P_{m+2}-P_{m}-P_{2}=\left(m^{2}+m\right) K_{X}^{3}-\chi\left(\mathcal{O}_{X}\right)+(l(m+2)-l(m)-l(2)) .
$$

By $\left[\mathbf{1 1}\right.$, Lemma 3.1], one sees $l(m+2)-l(m)-l(2) \geq 0$. Since $K_{X}^{3}>0$ and $\chi\left(\mathcal{O}_{X}\right)=1$, we have $P_{m+2}-P_{m}-P_{2}>-1$.

We consider the formal basket

$$
\mathbf{B}:=\left(B, \chi\left(\mathcal{O}_{X}\right), P_{2}(X)\right)
$$

where $B=\mathscr{B}(X)$. As we have seen in [4, Section 3],

(i) $K^{3}(\mathbf{B})=K^{3}(B)=K_{X}^{3}>0$;

(ii) $P_{m}(\mathbf{B})=P_{m}(X)$ for all $m \geq 2$.

By Lemma 3.2, we see $P_{4} \geq 2$ if $P_{2}>0$. Thus under Assumption 3.1, we have $P_{2}=0$. We can also get $P_{m+2}>0$ whenever $P_{m}>0$. Thus, in practice, we only need to study the following types: $P_{2}=0$ and

$$
\begin{aligned}
\left(P_{3}, P_{4}, P_{5}, P_{6}\right)= & (0,0,0,0),(0,0,0,1),(0,0,1,0),(0,0,1,1), \\
& (0,1,0,1),(0,1,1,1),(1,0,1,1),(1,1,1,1) .
\end{aligned}
$$

Now we consider the formal basket $\mathbf{B}:=(B, 1,0)$. We might abuse the notation of baskets and formal baskets in this section for we always have $\chi=1, P_{2}=0$ in this section. We keep the notation as in [4].

With explicit values of $\left(P_{3}, P_{4}, P_{5}, P_{6}\right)$, we are able to determine $\mathscr{B}^{(5)}(B)$ (cf. [4, Sections 3,4]). Our main task is to search all possible minimal (with regard to $\succ$ ) positive baskets $B_{\text {min }}$ dominated by $\mathscr{B}^{(5)}(B)$. Take $\mathbf{B}^{\mathbf{5}}:=\left(\mathscr{B}^{(5)}(B), 1,0\right)$ and $\mathbf{B}_{\text {min }}:=\left(B_{\text {min }}, 1,0\right)$. Then we see $\mathbf{B}^{\mathbf{5}} \succcurlyeq \mathbf{B} \succcurlyeq \mathbf{B}_{\text {min }}$.

Now we classify all minimal positive geometric baskets $B_{\min }$.

3.3. Case I: $P_{3}=P_{4}=P_{5}=P_{6}=0$ (impossible)

We have $\sigma=10, \tau=4, \Delta^{3}=5, \Delta^{4}=14, \epsilon=0, \sigma_{5}=0$, and $\epsilon_{5}=2$. The only possible initial basket is $\{5 \times(1,2), 4 \times(1,3),(1,4)\}$. And 
$B^{(5)}=\{3 \times(1,2), 2 \times(2,5), 2 \times(1,3),(1,4)\}$ with $K^{3}=\frac{1}{60}$. We shall calculate $B_{\min }$ of $B^{(5)}$.

If we pack $\{(1,2),(2,5)\}$ into $\{(3,7)\}$. Then we get:

I-1. $B_{1,1}=\{2 \times(1,2),(3,7),(2,5), 2 \times(1,3),(1,4)\}, K^{3}=\frac{1}{420}$,

which admits no further prime packing into positive baskets. Hence $B_{1,1}$ is minimal positive.

We consider those baskets with $(1,2)$ unpacked because otherwise it's dominated by $B_{1,1}$. So we consider the packing

$$
\{3 \times(1,2),(2,5),(3,8),(1,3),(1,4)\}
$$

with $K^{3}=\frac{1}{120}$. This basket allows two further packings to minimal positive ones:

I-2. $B_{1,2}=\{3 \times(1,2),(2,5),(4,11),(1,4)\}, K^{3}=\frac{1}{220}$.

I-3. $B_{1,3}=\{3 \times(1,2),(5,13),(1,3),(1,4)\}, K^{3}=\frac{1}{156}$.

Finally we consider the case that both $(1,2)$ and $(2,5)$ remain unpacked. We get one more basket which is indeed minimal positive

I-4. $B_{1,4}=\{3 \times(1,2), 2 \times(2,5),(1,3),(2,7)\}, K^{3}=\frac{1}{210}$.

A direct calculation shows that none of $B_{1,1}, B_{1,2}, B_{1,3}$, and $B_{1,4}$ satisfy inequality (2.7). Hence $B$ does not satisfy (2.7), a contradiction. This proves that Case I is impossible.

3.4. Case II: $P_{3}=P_{4}=P_{5}=0, P_{6}=1\left(\Rightarrow B_{2,1}, B_{2,2}\right)$

Now we have $\sigma=10, \tau=4, \Delta^{3}=5, \Delta^{4}=14, \epsilon \leq 1$. If $\epsilon=0$, then $\epsilon_{5}=1$; if $\epsilon=1$, then $\epsilon_{5}=0$. Thus all possible initial baskets and $B^{(5)}$ are as follows:

II-i. $B^{(0)}=\{5 \times(1,2), 4 \times(1,3),(1,4)\} \succ B^{(5)}=\{4 \times(1,2),(2,5), 3 \times$ $(1,3),(1,4)\}$, with $K^{3}\left(B^{(5)}\right)=\frac{1}{20}$.

II-ii. $B^{(0)}=\{5 \times(1,2), 4 \times(1,3),(1,5)\} \succ B^{(5)}=\{5 \times(1,2), 4 \times$ $(1,3),(1,5)\}$, with $K^{3}\left(B^{(5)}\right)=\frac{1}{30}$.

In Case II-i, we first consider the situation that all single baskets $(1,2)$ are packed into $\{(6,13), 3 \times(1,3),(1,4)\}$, which gives a unique minimal positive basket:

II-1. $B_{2,1}=\{(6,13),(1,3),(3,10)\}, K^{3}=\frac{1}{390}, P_{9}=2, P_{13}=3$.

We then consider the situation that at least one basket $(1,2)$ remains unpacked. Then we get the following minimal positive basket:

II-2. $B_{2,2}=\{(1,2),(5,11),(4,13)\}, K^{3}=\frac{1}{286}, P_{9}=2, P_{13}=3$.

Notice, however, that if $\{3 \times(1,2),(3,7), 3 \times(1,3),(1,4)\} \succ B$, then $B$ dominates $B_{2,2}$. Thus it remains to consider the situation that all single baskets $(1,2)$ are unpacked, but $(2,5)$ must be packed with some $(1,3)$. So we get the following minimal positive baskets:

II-3. $B_{2,3}=\{(4,8),(3,8),(3,10)\}, K^{3}=\frac{1}{40}$.

II-4. $B_{2,4}=\{(4,8),(4,11),(2,7)\}, K^{3}=\frac{2}{77}$. 
II-5. $B_{2,5}=\{(4,8),(5,14),(1,4)\}, K^{3}=\frac{1}{28}$.

In Case II-ii, $B^{(5)}$ admits no further prime packing. Thus we get:

II-6. $B_{2,6}=\{(5,10),(4,12),(1,5)\}, K^{3}=\frac{1}{30}$.

One may check that $B_{2,3}, B_{2,4}, B_{2,5}, B_{2,6}$ do not satisfy inequality (2.7). Thus only II-1 and II-2 can happen.

3.5. Case III: $P_{3}=P_{4}=0, P_{5}=1, P_{6}=0\left(\Rightarrow B_{3,1} \sim B_{3,5}\right)$

Now we have $\sigma=10, \tau=4, \Delta^{3}=5, \Delta^{4}=15$. Moreover, $P_{7} \geq 1$, and hence $\epsilon=0, \sigma_{5}=0$, and $\epsilon_{5}=4$. Thus the only possible initial basket and $B^{(5)}$ are

$$
B^{(0)}=\{5 \times(1,2), 5 \times(1,3)\} \succ B^{(5)}=\{(1,2), 4 \times(2,5),(1,3)\} .
$$

So we get the following minimal positive baskets:

III-1. $B_{3,1}=\{(9,22),(1,3)\}, K^{3}=\frac{1}{66}, P_{9}=2, P_{10}=3$.

III-2. $B_{3,2}=\{(7,17),(3,8)\}, K^{3}=\frac{1}{136}, P_{10}=2, P_{12}=3$.

III-3. $B_{3,3}=\{(5,12),(5,13)\}, K^{3}=\frac{1}{156}, P_{10}=2, P_{12}=3$.

III-4. $B_{3,4}=\{(3,7),(7,18)\}, K^{3}=\frac{1}{126}, P_{10}=2, P_{12}=3$.

III-5. $B_{3,5}=\{(1,2),(9,23)\},, K^{3}=\frac{1}{46}, P_{8}=2, P_{10}=4$.

3.6. Case IV: $P_{3}=P_{4}=0, P_{5}=1, P_{6}=1\left(\Rightarrow B_{3,1}, B_{3,2}, B_{3,4}, B_{3,5}\right)$

Now we have $\sigma=10, \tau=4, \Delta^{3}=5, \Delta^{4}=15$. Moreover, the initial basket must have $n_{1,2}^{0}=n_{1,3}^{0}=5$, and hence $n_{1, r}^{0}=0$ for all $r \geq 4$. It follows that $\epsilon=0, \sigma_{5}=0$, and $\epsilon_{5}=3$. Thus the only possible initial basket and $B^{(5)}$ are

$B^{(0)}=\{5 \times(1,2), 5 \times(1,3)\} \succ B^{(5)}=\{2 \times(1,2), 3 \times(2,5), 2 \times(1,3)\}$.

So we get the following minimal positive baskets:

IV-1. $\{(8,19),(2,6)\} \succ B_{3,1}$.

IV-2. $\{(6,14),(4,11)\} \succ B_{3,4}$.

IV-3. $\{(4,9),(6,16)\} \succ B_{3,2}$.

IV-4. $\{(2,4),(8,21)\} \succ B_{3,5}$.

3.7. Case V: $P_{3}=0, P_{4}=1, P_{5}=0, P_{6}=1$. $\left(\Rightarrow B_{5,1} \sim B_{5,3}\right)$

We have $\sigma=10, \tau=4, \Delta^{3}=6, \Delta^{4}=13$, and $\sigma_{5} \leq \epsilon \leq 2$. The initial baskets have 4 types:

V-i. $\{6 \times(1,2),(1,3), 3 \times(1,4)\}$;

V-ii. $\{6 \times(1,2),(1,3), 2 \times(1,4),(1,5)\}$;

V-iii. $\{6 \times(1,2),(1,3),(1,4), 2 \times(1,5)\}$;

V-iv. $\{6 \times(1,2),(1,3), 2 \times(1,4),(1, r)\}$ with $r \geq 6$.

Cases V-iii and V-iv are impossible since $K^{3} \leq 0$. For Case V-i, we have $\epsilon_{5}=1$, and for $\mathrm{V}$-ii, we have $\epsilon_{5}=0$. Hence $B^{(5)}$ has two possibilities, correspondingly: 
V-i. $\{(5,10),(2,5),(3,12)\}$;

V-ii. $\{(6,12),(1,3),(2,8),(1,5)\}$.

By computation, we get minimal positive baskets as follows:

V-1. $B_{5,1}=\{(7,15),(3,12)\}, K^{3}=\frac{1}{60}, P_{7}=2, P_{8}=3$.

V-2. $B_{5,2}=\{(6,12),(1,3),(3,13)\}, K^{3}=\frac{1}{39}, P_{8}=3$.

V-3. $B_{5,3}=\{(6,12),(3,11),(1,5)\}, K^{3}=\frac{1}{55}, P_{8}=2, P_{10}=4$.

3.8. Case VI: $P_{3}=0, P_{4}=P_{5}=P_{6}=1\left(\Rightarrow B_{6,1} \sim B_{6,6}\right)$

We have $\sigma=10, \tau=4, \Delta^{3}=6, \Delta^{4}=14$. Also, $P_{7} \geq 1$ and hence $\sigma_{5} \leq \epsilon \leq 2$. The initial baskets have four types:

VI-i. $\{6 \times(1,2), 2 \times(1,3), 2 \times(1,4)\}$;

VI-ii. $\{6 \times(1,2), 2 \times(1,3),(1,4),(1,5)\}$;

VI-iii. $\{6 \times(1,2), 2 \times(1,3), 2 \times(1,5)\}$;

VI-iv. $\{6 \times(1,2), 2 \times(1,3),(1,4),(1, r)\}$ with $r \geq 6$.

Since there are only 2 baskets of $(1,3)$, we have $\epsilon_{5}=3-\sigma_{5} \leq 2$.

Hence $\sigma_{5}>0$ and $\epsilon>0$. Therefore, Case VI-i is impossible.

For Case VI-ii and $\epsilon_{5}=2$, we get:

VI-ii. $B^{(5)}=\{4 \times(1,2), 2 \times(2,5),(1,4),(1,5)\}$.

Hence we get minimal positive baskets as follows:

VI-1. $B_{6,1}=\{(1,2),(7,16),(2,9)\}, K^{3}=\frac{1}{144}, P_{7}=2, P_{9}=3$.

VI-2. $B_{6,2}=\{(6,13),(2,5),(2,9)\}, K^{3}=\frac{8}{585}, P_{7}=2, P_{8}=3$.

VI-3. $B_{6,3}=\{(8,18),(1,4),(1,5)\}, K^{3}=\frac{1}{180}, P_{7}=2, P_{9}=3$.

For Case VI-iii and $\epsilon_{5}=1$, we get:

VI-ii. $B^{(5)}=\{5 \times(1,2),(2,5),(1,3), 2 \times(1,5)\}$.

Hence we get minimal positive baskets as follows:

VI-4. $B_{6,4}=\{(1,2),(6,13),(1,3),(2,10)\}, K^{3}=\frac{1}{390}, P_{8}=2, P_{9}=3$.

VI-5. $B_{6,5}=\{(5,10),(3,8),(2,10)\}, K^{3}=\frac{1}{40}, P_{8}=3$.

For Case VI-iv and $\epsilon_{5}=2$, we get:

VI-iv. $B^{(5)}=\{4 \times(1,2), 2 \times(2,5),(1,4),(1, r)\}$ with $r \geq 6$.

Since $K^{3}\left(B^{(5)}\right)>0$, we must have $r=6$. Then we get the following minimal positive basket:

VI-6. $B_{6,6}=\{(3,6),(3,7),(2,5),(1,4),(1,6)\}, K^{3}=\frac{1}{420}, P_{10}=2$, $P_{12}=3$.

3.9. Case VII: $P_{3}=1, P_{4}=0, P_{5}=P_{6}=1$ (impossible)

We have $\sigma=9, \tau=3, \Delta^{3}=1, \Delta^{4}=9$. Moreover, $P_{7} \geq 1$ and hence $\epsilon=0$. It follows that $\sigma_{5}=0$ and $\epsilon_{5}=2$. The initial basket is $B^{(0)}=\{(1,2), 7 \times(1,3),(1,4)\}$.

Note that there is only one basket of type $(1,2)$. However, since $\epsilon_{5}=2$, one has $1 \geq n_{2,5}^{5}=2$, a contradiction. Thus Case VII does not happen. 
3.10. Case VIII: $P_{3}=P_{4}=P_{5}=P_{6}=1\left(\Rightarrow B_{8,1} \sim B_{8,3}\right)$

We have $\sigma=9, \tau=3, \Delta^{3}=2, \Delta^{4}=8$. Moreover, $P_{7} \geq 1$ and then $\epsilon \leq 1$. If $\epsilon=1$, then $\sigma_{5}=1$ and $\epsilon_{5}=1$. If $\epsilon=0$, then $\sigma_{5}=0$ and $\epsilon_{5}=2$. The initial baskets and $B^{(5)}$ have 2 types:

VIII-i. $B^{(0)}=\{2 \times(1,2), 4 \times(1,3), 3 \times(1,4)\} \succ B^{(5)}=\{2 \times(2,5), 2 \times$ $(1,3), 3 \times(1,4)\}$ with $K^{3}\left(B^{(5)}\right)=\frac{1}{60}$.

VIII-ii. $B^{(0)}=\{2 \times(1,2), 4 \times(1,3), 2 \times(1,4),(1,5)\} \succ B^{(5)}=\{(1,2)$, $(2,5), 3 \times(1,3), 2 \times(1,4),(1,5)\}$ with $K^{3}\left(B^{(5)}\right)=0$.

Clearly, Case VIII-ii is impossible since $K^{3}$ is not positive.

For Case VIII-i, we first consider the situation that one single basket $(2,5)$ is packed, so that we get the basket $\{(2,5),(3,8),(1,3),(4,12)\}$. We can get two minimal positive baskets as follows:

VIII-1. $B_{8,1}=\{(5,13),(1,3),(3,12)\}, K^{3}=\frac{1}{156}, P_{7}=2, P_{8}=3$.

VIII-2. $B_{8,2}=\{(2,5),(4,11),(3,12)\}, K^{3}=\frac{1}{220}, P_{7}=2, P_{8}=3$.

It remains to consider the situation that each single basket $(2,5)$ remains unpacked. We then obtain the basket

$$
B_{210}:=\{(4,10),(1,3),(2,7),(2,8)\}
$$

with $K^{3}=\frac{1}{210}, P_{7}=2, P_{10}=3$. After a one-step prime packing, we get the minimal positive basket:

VIII-3. $B_{8,3}=\{(4,10),(1,3),(3,11),(1,4)\}, K^{3}=\frac{1}{660}, P_{7}=2$.

The detailed classification $(3.3 \sim 3.10)$ makes it possible for us to study the birational geometry of $X$, of which the first application is the following theorem.

Theorem 3.11. Assume $\chi\left(\mathcal{O}_{X}\right)=1$. Then $K_{X}^{3} \geq \frac{1}{420}$. Furthermore, $K_{X}^{3}=\frac{1}{420}$ if, and only if, $\mathscr{B}=B_{6,6}$.

Proof. If $\mu_{1} \leq 6$, then Proposition 2.30 implies $K_{X}^{3} \geq \frac{1}{294} \cdot \frac{11}{12}>\frac{1}{420}$.

We may assume that $P_{m} \leq 1$ for $m \leq 6$. We have seen $P_{2}=0$. Since $\mathbf{B}^{\mathbf{5}} \succcurlyeq \mathbf{B} \succcurlyeq \mathbf{B}_{\min }$ and by [4, Lemma 3.6], we have

$$
K_{X}^{3}=K^{3}(B) \geq K^{3}\left(B_{\min }\right)
$$

where $B_{\text {min }}$ is in the set $\left\{B_{2,1}, B_{2,2}, B_{3,1} \sim B_{3,5}, B_{5,1} \sim B_{5,3}, B_{6,1} \sim\right.$ $\left.B_{6,6}, B_{8,1} \sim B_{8,3}\right\}$.

If $B_{\min } \neq B_{6,6}$, or $B_{8,3}$, then we have seen $K^{3}\left(B_{\min }\right)>\frac{1}{420}$.

If $B_{\text {min }}=B_{8,3}$, we show $B \neq B_{8,3}$. In fact, if $B=B_{8,3}$, then $P_{7}(B)=2$ as we have seen in 3.10. By Table $\mathrm{A}$ in Section 2, we have $K_{X}^{3}=K^{3}(\mathbf{B}) \geq \frac{5}{2408}>\frac{1}{660}$, a contradiction. Hence $B \succ B_{8,3}$. Notice that $B_{8,3}$ is obtained, exactly, by one-step packing from

$$
B_{210}:=\{(4,10),(1,3),(2,7),(2,8)\}
$$

and no other ways. This says $B \succcurlyeq B_{210}$ and so $K_{X}^{3} \geq K^{3}\left(B_{210}\right)=\frac{1}{210}$.

We have seen $K^{3}\left(B_{6,6}\right)=\frac{1}{420}$. We are done. 
With a different approach, L. Zhu $[\mathbf{2 9}]$ also proved $K^{3} \geq \frac{1}{420}$. The proof of the last theorem gives the following:

Corollary 3.12. Assume $\chi\left(\mathcal{O}_{X}\right)=1$ and $P_{m} \leq 1$ for all $m \leq 6$. Then $\mathscr{B}(X)$ either dominates a minimal basket in the set

$$
\left\{B_{2,1}, B_{2,2}, B_{3,1} \sim B_{3,5}, B_{5,1} \sim B_{5,3}, B_{6,1} \sim B_{6,6}, B_{8,1}, B_{8,2}\right\}
$$

or dominates the basket $B_{210}$.

Corollary 3.13. Assume $\chi\left(\mathcal{O}_{X}\right)=1$. Then $P_{10}(X) \geq 2$ and, in particular, $\mu_{1} \leq 10$.

Proof. If $P_{m_{0}} \geq 2$ for some $m_{0} \leq 6$, then, by Lemma 3.2, one can see $P_{10} \geq 2$. Otherwise, Corollary 3.12 and [4, Lemma 3.6] imply that $P_{10}=P_{10}(\mathscr{B}(X)) \geq P_{10}\left(B_{*}\right)$ where $B_{*}$ denotes a minimal positive basket mentioned in Corollary 3.12. By a direct computation, we get $P_{10}\left(B_{*}\right) \geq 2$.

q.e.d.

Example 1.3 shows that the statement in Corollary 3.13 is optimal since $P_{9}\left(X_{46}\right)=1$.

Theorem 3.14. Assume $\chi\left(\mathcal{O}_{X}\right)=1$. Then:

(1) $\rho_{0} \leq 7$.

(2) Either $P_{5}>0$ or $P_{6}>0$.

Proof. (1) Recall that $\mu_{0}:=\min \left\{m \mid P_{m}>0\right\}$. By 3.3, we see $\mu_{0} \leq 6$. When $\mu_{0} \leq 3$, it is easy to deduce the statement by Lemma 3.2.

When $\mu_{0}=4$, Lemma 3.2 implies $P_{2 k}>0$ for all $k \geq 3$. If $P_{7}>0$, Lemma 3.2 implies $P_{2 k+1}>0$ for all $k \geq 3$ and the statement (1) is true. Assume $P_{7}=0$. Then $P_{5}=0$. Now $\epsilon_{5}=2-P_{6}-\sigma_{5} \geq 0$ implies $\sigma_{5} \leq 2-P_{6} \leq 1$. On the other hand, $\epsilon_{6}=P_{4}+P_{6}-\epsilon=0$ implies $\epsilon \geq 2$. This means $\sigma_{5}=P_{6}=P_{4}=1$ and the situation corresponds to 3.7. Thus $B \succcurlyeq B_{\min }$ where $B_{\min }=B_{5,2}, B_{5,3}$. But the computation tells $P_{7}\left(B_{\min }\right)>0$, a contradiction.

When $\mu_{0}=5$, we study $P_{8}$. If $P_{8}>0$, then (1) is true by Lemma 3.2. Assume $P_{8}=0$. Then $P_{6}=0$. Now $\epsilon_{6}=P_{5}-P_{7}-\epsilon=0$ gives $\epsilon=0$ and $P_{5}=P_{7}$ since $P_{7} \geq P_{5}$. Since $n_{1,4}^{0}=1-P_{5} \geq 0$, we see $P_{5}=1$. So the situation corresponds to 3.5. Since the computation shows $P_{8} \geq P_{8}\left(B_{3, *}\right)>0$, a contradiction.

Finally, when $\mu_{0}=6$, we study $P_{7}$. If $P_{7}>0$, then Lemma 3.2 implies (1). Otherwise, $P_{7}=0$. Now $\epsilon_{6}=P_{6}-\epsilon=0$ implies $\epsilon=P_{6}>0$. Besides, $\epsilon_{5}=2-P_{6}-\sigma_{5} \geq 0$ says $P_{6} \leq 1$ since $\sigma_{5}>0$. Hence $\epsilon=P_{6}=1$. The situation corresponds to 3.4. But the computation shows $P_{7} \geq P_{7}\left(B_{2,1}\right)>0$ or $P_{7} \geq P_{7}\left(B_{2,2}\right)>0$, a contradiction.

(2) Assume $P_{5}=P_{6}=0$. Then Lemma 3.2 implies $P_{3}=P_{4}=0$. The situation corresponds to 3.3 , which is impossible as already seen there. q.e.d.

D. Shin $[\mathbf{2 2}]$ proved the first statement in a different way. 


\section{General type 3-folds with $\chi>1$}

In this section, we assume $\chi\left(\mathcal{O}_{X}\right)>1$. Again, we will frequently apply our formulae and inequalities in [4, Sections 3 and 4].

When $P_{m_{0}} \geq 2$ for some positive integer $m_{0} \leq 12$, known theorems will give an effective lower bound of $K_{X}^{3}$ and a practical pluricanonical birationality. Therefore, similar to Section 3, we need to classify $X$ up to baskets when preceding plurigenera are smaller. For this reason, we make the following:

4.1. Assumption: $P_{m} \leq 1$ for all $m \leq 12$.

According to $\left[\mathbf{4}\right.$, Lemma 4.8], we have seen that $P_{2}=0$ under Assumption 4.1. Note that inequality $[\mathbf{4},(3.14)]$, for general-type 3 -folds, is as follows:

$$
2 P_{5}+3 P_{6}+P_{8}+P_{10}+P_{12} \geq \chi+10 P_{2}+4 P_{3}+P_{7}+P_{11}+P_{13}+R
$$

where

$$
\begin{aligned}
R:= & 14 \sigma_{5}-12 n_{1,5}^{0}-9 n_{1,6}^{0}-8 n_{1,7}^{0}-6 n_{1,8}^{0}-4 n_{1,9}^{0}-2 n_{1,10}^{0}-n_{1,11}^{0} \\
= & 2 n_{1,5}^{0}+5 n_{1,6}^{0}+6 n_{1,7}^{0}+8 n_{1,8}^{0}+10 n_{1,9}^{0}+12 n_{1,10}^{0}+13 n_{1,11}^{0} \\
& +14 \sum_{r \geq 12} n_{1, r}^{0}
\end{aligned}
$$

and $\sigma_{5}=\sum_{r>5} n_{1, r}^{0}$.

Inequality (4.1) and Assumption 4.1 imply that both $\chi$ and $P_{13}$ are bounded from above. Thus our formulae in [4, Section 4] allows us to explicitly compute $B^{(12)}$. To be more solid, we prove the following:

Proposition 4.2. Assume $\chi\left(\mathcal{O}_{X}\right)>1$ and $P_{m} \leq 1$ for all $m \leq 12$. Then the formal basket $\mathbf{B}=\mathbf{B}(X):=\left(\mathscr{B}(X), \chi\left(\mathcal{O}_{X}\right), 0\right)$ has a finite number of possibilities.

Proof. We study $n_{1, r}^{0}$ for $r \geq 6$. If there exists a number $r \geq 6$ such that $n_{1, r}^{0} \neq 0$, then $R \geq 5$ by the definition of $R$ in inequality (4.1). Hence, by (4.1), one has

$$
8 \geq 2 P_{5}+3 P_{6}+P_{8}+P_{10}+P_{12} \geq \chi+5 \geq 7 .
$$

This implies that $P_{5}=P_{6}=1$. Hence $P_{11}=1$. Now (4.1) again reads $5+P_{8}+P_{10}+P_{12} \geq 8+P_{7}+P_{13}$. It follows that $P_{8}=P_{10}=P_{12}=1$ and $P_{7}=P_{13}=0$. This gives a contradiction since $P_{13} \geq P_{5} P_{8}=1$. So we conclude $n_{1, r}^{0}=0$ for all $r \geq 6$. In other words, [4, Assumption 3.8] is satisfied.

This essentially allows us to utilize those formulae in the last part of [4, Section 3]. In particular, one sees that each quantity there is bounded and hence $B^{(12)}$ has a finite number of possibilities. Dominated by $B^{(12)}$ (i.e., $\left.B^{(12)} \succcurlyeq B\right), B=\mathscr{B}(X)$ also has a finite number of possibilities. We are done. 
4.3. Complete classification of B satisfying Assumption 4.1.

Note that, for all $0<m, n \leq 12$, and $m+n \leq 13$,

$$
P_{m+n} \geq P_{m} P_{n}
$$

naturally holds since $P_{m}, P_{n} \leq 1$.

Suppose we have known $B^{(12)}$. Then we can determine all possible minimal positive baskets $B_{\min }$ dominated by $B^{(12)}$, where $B_{\min } \in T$ (a finite set). Now the formal basket $\mathbf{B}$ satisfies the following relation:

$$
\left(B^{(12)}, \chi, 0\right) \succcurlyeq \mathbf{B} \succcurlyeq\left(B_{\min }, \chi, 0\right)
$$

for some $B_{\min } \in T$. Therefore, by [4, Lemma 3.6], we have $K_{X}^{3}=$ $K^{3}(B) \geq K^{3}\left(B_{\min }\right)>0$ and $P_{m}=P_{m}(B) \geq P_{m}\left(B_{\min }\right)$. This is the whole strategy.

The calculation can be done by a simple computer program, or even by hand. Our main result is Table $\mathrm{C}$, which is a complete list of all possibilities of $B^{(12)}$ and its minimal positive elements.

In fact, first we preset $P_{m}=0,1$ for $m=3, \cdots, 11$. Then $\epsilon_{6}=0$ gives the value of $\epsilon$. So we know the value of $n_{1,5}^{0}$. By inequality (4.1) we get the upper bound of $\chi$ since $P_{13} \geq 0$. Since $n_{1,4}^{7} \geq 0$, we get the upper bound of $\eta$. Similarly $n_{2,9}^{9} \geq 0$ gives the upper bound of $\zeta$. Also $n_{4,9}^{11} \geq 0$ yields $\alpha \leq \zeta$. Finally, $n_{3,8}^{11} \geq 0$ gives the upper bound of $\beta$. Now we set $P_{12}=0,1$. Then inequality (4.1) again gives the upper bound of $P_{13}$, noting that $\chi \geq 2$. Clearly there are, at most, finitely many solutions. With inequality (4.2) imposed, we can get about 80 cases. An important property to mention is the inequality $K^{3}\left(B^{(12)}\right) \geq K^{3}(B)=K_{X}^{3}>0$. With $K^{3}>0$ imposed on, we have 63 outputs, which is exactly Table C. Simultaneously, we have been able to calculate all those minimal positive baskets dominated by $B^{(12)}$, since $B^{(12)}$ is "nearly" minimal in most cases.

If one would like to take a direct calculation by hand, it is of course possible. Consider the no. 2 case in Table $\mathrm{C}$ as an example. Since $P_{2}=0, P_{3}=\cdots=P_{7}=0, P_{8}=1$, and $P_{9}=P_{10}=P_{11}=0,[\mathbf{4},(3.10)]$ tells that $\epsilon=0$ and thus $\sigma_{5}=0$, which means $R=0$. Now inequality (4.1) gives $P_{12}+1 \geq \chi+P_{13} \geq 2$. So $P_{12}=1, \chi=2$, and $P_{13}=0$. Now the formula for $\epsilon_{10}$ gives $\epsilon_{10}=-\eta \geq 0$, which means $\eta=0$. Similarly, $n_{1,5}^{9}=\zeta-1 \geq 0$. On the other hand, $n_{3,7}^{9}=1-\zeta \geq 0$. Thus $\zeta=1$. Now $n_{4,9}^{11}=\zeta-\alpha \geq 0$ gives $\alpha \leq 1$. $n_{3,11}^{11}=1-\zeta-\alpha-\beta \geq$ gives $\alpha=\beta=0$. Finally, we get

$$
\left\{n_{1,2}, n_{5,12}, \ldots, n_{1,5}\right\}=\{4,0,1,0,0,2,1,0,3,0,0,0,2,0,0\} .
$$

That is, $B^{(12)}=\{4 \times(1,2),(4,9), 2 \times(2,5),(3,8), 3 \times(1,3), 2 \times(1,4)\}$.

We see that $B^{(12)}$ admits only one prime packing of type

$$
\{(2,5),(3,8)\} \succ\{(5,13)\}
$$


over the minimal positive basket $\{4 \times(1,2),(4,9),(2,5),(5,13), 3 \times(1,3), 2 \times$ $(1,4)\}$. We simply write this as $\{(5,13), *\}$ in Table C. It is now easy to calculate $K^{3}$ for both $B^{(12)}$ and the minimal positive basket $\{(5,13), *\}$. Finally, we can directly calculate $P_{m}$. At the same time, $\mu_{1}$ is given in the table. For our needs in this context, we also display the value of $P_{18}=P_{18}\left(B^{(12)}\right)$ or $P_{18}\left(B_{\min }\right)$ and $P_{24}=P_{24}\left(B^{(12)}\right)$ or $P_{24}\left(B_{\min }\right)$ in Table $\mathrm{C}$, though the symbols $P_{18}$ or $P_{24}$ are misused here.

So theoretically we can finish our classification by detailed computations. We omit the details because all calculations are similar.

4.4. Notation. By abuse of notation, we denote by $B_{*}$ the final basket corresponding to No.* in Table C. For example, $B_{2}=\{4 \times$ $(1,2),(4,9), 2 \times(2,5),(3,8), 3 \times(1,3), 2 \times(1,4)\}$ while $B_{2 a}=\{4 \times$ $(1,2),(4,9),(2,5),(5,13), 3 \times(1,3), 2 \times(1,4)\}$ is minimal positive. The relation is as follows:

$$
B_{2} \succcurlyeq B \succcurlyeq B_{2 a} \text {. }
$$

Clearly, for this case, we have $\frac{1}{360}=K^{3}\left(B_{2}\right) \geq K_{X}^{3} \geq K^{3}\left(B_{2 a}\right)=\frac{1}{1170}$.

Another typical example is No.63, where we have

$B_{63}=\{5 \times(1,2),(4,9), 2 \times(3,7),(2,5),(3,8),(4,11), 3 \times(1,3),(2,7),(1,5)\}$, which is already minimal positive. So we have the relation

$$
B^{(12)}=B_{63}=B=B_{\text {min }}
$$

and thus $K_{X}^{3}=\frac{1}{5544}$. Of course, we will see that No.63 does not happen on any $X$.

Now we begin to analyze Table $\mathrm{C}$ and pick out "impossible" cases.

Proposition 4.5. In Table $C, B \neq B_{*}$ for any $B_{*}$ in the set

$$
\begin{aligned}
& \left\{B_{4 a}, B_{9}, B_{16 a}, B_{16 c}, B_{18 a}, B_{20 a}, B_{21 a}, B_{22}, B_{24}, B_{27 a},\right. \\
& \left.B_{29 a}, B_{33 a}, B_{44 b}, B_{46 a}, B_{47}, B_{52 a}, B_{55}, B_{60 a}, B_{61}, B_{63}\right\} .
\end{aligned}
$$

In particular, cases No. 9, No. 22, No. 24, No. 47, No. 55, No. 61, and No. 63 do not happen at all. 
Table C

\begin{tabular}{|c|c|c|c|c|c|c|c|}
\hline No. & $\left(P_{3}, \cdots, P_{11}\right)$ & $P_{18}$ & $P_{24}$ & $\mu_{1}$ & $\chi$ & $B^{(12)}=\left(n_{1,2}, n_{5,11}, \cdots, n_{1,5}\right)$ or $B_{\min }$ & $K^{3}$ \\
\hline 1 & $(0,0,0,0,0,0,0,1,0)$ & 4 & 8 & 14 & 2 & $(5,0,0,1,0,3,0,0,3,0,0,1,0,0,0)$ & $\frac{3}{770}$ \\
\hline 2 & $(0,0,0,0,0,1,0,0,0)$ & 3 & 7 & 15 & 2 & $(4,0,1,0,0,2,1,0,3,0,0,0,2,0,0)$ & $\frac{1}{360}$ \\
\hline $2 a$ & & 2 & 3 & 18 & & $\{(2,5),(3,8), *\} \succ\{(5,13), *\}$ & $\frac{1}{1170}$ \\
\hline 3 & $(0,0,0,0,0,1,0,1,0)$ & 3 & 7 & 15 & 3 & $(6,1,0,0,0,4,1,0,4,0,1,0,2,0,0)$ & $\frac{23}{9240}$ \\
\hline $3 a$ & & 2 & 3 & 18 & & $\{(2,5),(3,8), *\} \succ\{(5,13), *\}$ & $\frac{17}{30030}$ \\
\hline 4 & $(0,0,0,0,0,1,0,1,0)$ & 4 & 9 & 14 & 3 & $(7,0,1,0,0,4,0,1,3,0,1,0,2,0,0)$ & $\frac{13}{3465}$ \\
\hline $4 a$ & & 1 & 2 & 14 & & $\{(4,11),(2,6), *\} \succ\{(6,17), *\}$ & $\frac{1}{5355}$ \\
\hline 5 & $(0,0,0,0,0,1,0,1,0)$ & 5 & 10 & 14 & 3 & $(7,0,1,0,0,4,1,0,4,0,0,1,1,0,0)$ & $\frac{17}{3960}$ \\
\hline $5 a$ & & 4 & 3 & 15 & & $\{(8,20),(3,8), *\} \succ\{(11,28), *\}$ & $\frac{1}{1386}$ \\
\hline $5 b$ & & 3 & 3 & 15 & & $\{(5,13),(4,15), *\}$ & $\frac{1}{1170}$ \\
\hline 6 & $(0,0,0,1,0,0,0,1,0)$ & 3 & 6 & 14 & 3 & $(9,0,0,2,0,1,0,1,4,0,2,0,0,0,1)$ & $\frac{1}{462}$ \\
\hline 7 & $(0,0,0,1,0,0,1,0,0)$ & 3 & 5 & 14 & 2 & $(5,0,1,1,0,0,0,0,5,0,1,0,0,0,1)$ & $\frac{1}{630}$ \\
\hline $7 a$ & & 2 & 3 & 14 & & $\{(4,9),(3,7), *\} \succ\{(7,16), *\}$ & $\frac{10}{1680}$ \\
\hline 8 & $(0,0,0,1,0,0,1,1,0)$ & 3 & 5 & 14 & 3 & $(7,1,0,1,0,2,0,0,6,0,2,0,0,0,1)$ & $\frac{1}{770}$ \\
\hline 9 & $(0,0,0,1,0,1,0,0,0)$ & 2 & 2 & 14 & 3 & $(9,0,0,2,0,0,1,1,4,0,1,0,0,1,0)$ & $\frac{1}{5544}$ \\
\hline 10 & $(0,0,0,1,0,1,0,0,0)$ & 3 & 6 & 14 & 3 & $(8,0,1,1,0,0,2,0,5,0,1,0,1,0,1)$ & $\frac{1}{630}$ \\
\hline $10 a$ & & 2 & 4 & 14 & & $\{(4,9),(3,7), *\} \succ\{(7,16), *\}$ & $\frac{1}{1680}$ \\
\hline 11 & $(0,0,0,1,0,1,0,1,0)$ & 2 & 4 & 14 & 3 & $(9,0,0,2,0,0,1,1,3,1,0,0,1,0,1)$ & $\frac{3}{3080}$ \\
\hline $11 a$ & & 2 & 3 & 14 & & $\{(3,8),(4,11), *\} \succ\{(7,19), *\}$ & $\frac{1}{2660}$ \\
\hline 12 & $(0,0,0,1,0,1,0,1,0)$ & 5 & 11 & 14 & 3 & $(9,0,1,0,0,1,2,0,4,0,2,0,0,0,1)$ & $\frac{1}{252}$ \\
\hline $12 a$ & & 4 & 6 & 14 & & $\{(2,5),(6,16), *\} \succ\{(8,21), *\}$ & $\frac{1}{630}$ \\
\hline 13 & $(0,0,0,1,0,1,0,1,0)$ & 3 & 4 & 14 & 4 & $(12,0,0,2,0,2,0,2,4,0,2,0,0,1,0)$ & $\frac{4}{3465}$ \\
\hline 14 & $(0,0,0,1,0,1,0,1,0)$ & 3 & 6 & 14 & 4 & $(10,1,0,1,0,2,2,0,6,0,2,0,1,0,1)$ & $\frac{1}{770}$ \\
\hline 15 & $(0,0,0,1,0,1,0,1,0)$ & 4 & 8 & 14 & 4 & $(11,0,1,1,0,2,1,1,5,0,2,0,1,0,1)$ & $\frac{71}{27720}$ \\
\hline $15 a$ & & 2 & 4 & 14 & & $\{(4,11),(1,3), *\} \succ\{(5,14), *\}$ & $\frac{1}{2520}$ \\
\hline $15 b$ & & 3 & 4 & 14 & & $\{(2,5),(3,8), *\} \succ\{(5,13), *\}$ & $\frac{23}{36036}$ \\
\hline $15 c$ & & 3 & 5 & 14 & & $\{(7,16),(7,19), *\}$ & $\frac{31}{31920}$ \\
\hline 16 & $(0,0,0,1,0,1,0,1,0)$ & 5 & 9 & 14 & 4 & $(11,0,1,1,0,2,2,0,6,0,1,1,0,0,1)$ & $\frac{43}{13860}$ \\
\hline $16 a$ & & 4 & 3 & 14 & & $\{(4,10),(3,8), *\} \succ\{(7,18), *\}$ & $\frac{1}{3080}$ \\
\hline $16 b$ & & 4 & 4 & 14 & & $\{(2,5),(6,16), *\} \succ\{(8,21), *\}$ & $\frac{1}{1386}$ \\
\hline $16 c$ & & 3 & 3 & 14 & & $\{(7,16),(5,13), *\}$ & $\frac{3}{16016}$ \\
\hline 17 & $(0,0,0,1,0,1,0,1,1)$ & 3 & 6 & 14 & 3 & $(9,0,0,2,0,0,0,2,3,0,1,0,1,0,1)$ & $\frac{3}{1540}$ \\
\hline 18 & $(0,0,0,1,0,1,0,1,1)$ & 4 & 7 & 14 & 3 & $(9,0,0,2,0,0,1,1,4,0,0,1,0,0,1)$ & $\frac{23}{9240}$ \\
\hline $18 a$ & & 2 & 3 & 14 & & $\{(4,11),(1,3), *\} \succ\{(5,14), *\}$ & $\frac{1}{3080}$ \\
\hline $18 b$ & & 4 & 6 & 14 & & $\{(3,8),(4,11), *\} \succ\{(7,19), *\}$ & $\frac{83}{43890}$ \\
\hline 19 & $(0,0,0,1,0,1,1,0,0)$ & 3 & 3 & 14 & 3 & $(8,0,1,1,0,1,0,1,5,0,1,0,0,1,0)$ & $\frac{2}{3465}$ \\
\hline 20 & $(0,0,0,1,0,1,1,0,0)$ & 4 & 7 & 14 & 3 & $(7,0,2,0,0,1,1,0,6,0,1,0,1,0,1)$ & $\frac{1}{504}$ \\
\hline $20 a$ & & 3 & 3 & 18 & & $\{(2,5),(3,8), *\} \succ\{(5,13), *\}$ & $\frac{1}{16380}$ \\
\hline 21 & $(0,0,0,1,0,1,1,1,0)$ & 4 & 8 & 14 & 2 & $(6,0,1,0,0,0,1,0,3,1,0,0,0,0,1)$ & $\frac{1}{360}$ \\
\hline $21 a$ & & 2 & 3 & 16 & & $\{(1,3),(3,10), *\} \succ\{(4,13), *\}$ & $\frac{1}{4680}$ \\
\hline 22 & $(0,0,0,1,0,1,1,1,0)$ & 2 & 3 & 18 & 3 & $(7,1,0,1,0,1,1,0,5,1,0,0,1,0,1)$ & $\frac{1}{9240}$ \\
\hline 23 & $(0,0,0,1,0,1,1,1,0)$ & 3 & 5 & 14 & 3 & $(8,0,1,1,0,1,0,1,4,1,0,0,1,0,1)$ & $\frac{190}{13860}$ \\
\hline $23 a$ & & 2 & 3 & 14 & & $\{(4,9),(3,7), *\} \succ\{(7,16), *\}$ & $\frac{1}{2640}$ \\
\hline 24 & $(0,0,0,1,0,1,1,1,0)$ & 3 & 3 & 14 & 4 & $(10,1,0,1,0,3,0,1,6,0,2,0,0,1,0)$ & $\frac{1}{3465}$ \\
\hline 25 & $(0,0,0,1,0,1,1,1,0)$ & 4 & 7 & 14 & 4 & $(9,1,1,0,0,3,1,0,7,0,2,0,1,0,1)$ & $\frac{47}{27720}$ \\
\hline $25 a$ & & 4 & 6 & 14 & & $\{(5,11),(4,9), *\} \succ\{(9,20), *\}$ & $\frac{1}{840}$ \\
\hline 26 & $(0,0,0,1,0,1,1,1,0)$, & 5 & 9 & 14 & 4 & $(10,0,2,0,0,3,0,1,6,0,2,0,1,0,1)$ & $\frac{41}{13860}$ \\
\hline $26 a$ & & 3 & 5 & 14 & & $\{(4,11),(1,3), *\} \succ\{(5,14), *\}$ & $\frac{1}{1260}$ \\
\hline 27 & $(0,0,0,1,0,1,1,1,0)$ & 6 & 10 & 14 & 4 & $(10,0,2,0,0,3,1,0,7,0,1,1,0,0,1)$ & $\frac{97}{27720}$ \\
\hline $27 a$ & & 5 & 3 & 14 & & $\{(6,15),(3,8), *\} \succ\{(9,23), *\}$ & $\frac{19}{79695}$ \\
\hline $27 b$ & & 5 & 5 & 14 & & $\{(5,13),(5,18), *\}$ & $\frac{1}{1170}$ \\
\hline 28 & $(0,0,0,1,0,1,1,1,1)$ & 4 & 8 & 14 & 2 & $(5,1,0,0,0,0,1,0,4,0,1,0,0,0,1)$ & $\frac{230}{9240}$ \\
\hline 29 & $(0,0,0,1,0,1,1,1,1)$ & 5 & 10 & 14 & 2 & $(6,0,1,0,0,0,0,1,3,0,1,0,0,0,1)$ & $\frac{13}{3465}$ \\
\hline $29 a$ & & 2 & 3 & 14 & & $\{(4,11),(2,6), *\} \succ\{(6,17), *\}$ & $\frac{1}{5355}$ \\
\hline 30 & $(0,0,0,1,0,1,1,1,1)$ & 3 & 5 & 14 & 3 & $(7,1,0,1,0,1,0,1,5,0,1,0,1,0,1)$ & $\frac{1}{924}$ \\
\hline 31 & $(0,0,0,1,0,1,1,1,1)$ & 4 & 6 & 14 & 3 & $(7,1,0,1,0,1,1,0,6,0,0,1,0,0,1)$ & $\frac{1}{616}$ \\
\hline 32 & $(0,0,0,1,0,1,1,1,1)$ & 5 & 8 & 14 & 3 & $(8,0,1,1,0,1,0,1,5,0,0,1,0,0,1)$ & $\frac{2}{693}$ \\
\hline $32 a$ & & 4 & 6 & 14 & & $\{(4,9),(3,7), *\} \succ\{(7,16), *\}$ & $\frac{10}{528}$ \\
\hline $32 b$ & & 2 & 2 & 14 & & $\{(4,11),(1,3), *\} \succ\{(5,14), *\}$ & $\frac{1}{1386}$ \\
\hline 33 & $(0,0,0,1,1,0,0,1,0)$ & 2 & 4 & 14 & 2 & $(5,0,0,2,0,0,1,0,1,1,1,0,0,0,0)$ & $\frac{1}{840}$ \\
\hline $33 a$ & & 1 & 3 & 14 & & $\{(3,10),(2,7), *\} \succ\{(5,17), *\}$ & $\frac{1}{2856}$ \\
\hline
\end{tabular}




\begin{tabular}{|c|c|c|c|c|c|c|c|}
\hline No. & $\left(P_{3}, \cdots, P_{11}\right)$ & $P_{18}$ & $P_{24}$ & $\mu_{1}$ & $\chi$ & $\left(n_{1,2}, n_{4,9}, \cdots, n_{1,5}\right)$ or $B_{\min }$ & $K^{3}$ \\
\hline 34 & $(0,0,0,1,1,0,0,1,0)$ & 4 & 8 & 14 & 3 & $(7,0,1,1,0,2,1,0,3,0,3,0,0,0,0)$ & $\frac{1}{360}$ \\
\hline $34 a$ & & 3 & 6 & 14 & & $\{(4,9),(3,7), *\} \succ\{(7,16), *\}$ & $\frac{1}{560}$ \\
\hline $34 b$ & & 3 & 4 & 14 & & $\{(2,5),(3,8), *\} \succ\{(5,13), *\}$ & $\frac{1}{1170}$ \\
\hline 35 & $(0,0,0,1,1,0,0,1,1)$ & 3 & 6 & 14 & 2 & $(5,0,0,2,0,0,0,1,1,0,2,0,0,0,0)$ & $\frac{1}{462}$ \\
\hline 36 & $(0,0,0,1,1,0,1,1,0)$ & 3 & 5 & 14 & 2 & $(4,0,1,1,0,1,0,0,2,1,1,0,0,0,0)$ & $\frac{1}{630}$ \\
\hline $36 a$ & & 2 & 3 & 14 & & $\{(4,9),(3,7), *\} \succ\{(7,16), *\}$ & $\frac{1}{1680}$ \\
\hline $36 b$ & & 2 & 4 & 14 & & $\{(3,10),(2,7), *\} \succ\{(5,17), *\}$ & $\frac{1000}{5355}$ \\
\hline 37 & $(0,0,0,1,1,0,1,1,0)$ & 5 & 9 & 14 & 3 & $(6,0,2,0,0,3,0,0,4,0,3,0,0,0,0)$ & $\frac{1}{315}$ \\
\hline 38 & $(0,0,0,1,1,0,1,1,1)$ & 3 & 5 & 14 & 2 & $(3,1,0,1,0,1,0,0,3,0,2,0,0,0,0)$ & $\frac{1^{5}}{770}$ \\
\hline 39 & $(0,0,0,1,1,1,0,1,0)$ & 3 & 6 & 14 & 3 & $(7,0,1,1,0,1,2,0,2,1,1,0,1,0,0)$ & $\frac{10}{630}$ \\
\hline $39 a$ & & 2 & 4 & 14 & & $\{(4,9),(3,7), *\} \succ\{(7,16), *\}$ & $\frac{1}{1680}$ \\
\hline $39 b$ & & 2 & 5 & 14 & & $\{(3,10),(2,7), *\} \succ\{(5,17), *\}$ & $\frac{100}{5355}$ \\
\hline 40 & $(0,0,0,1,1,1,0,1,0)$ & 5 & 10 & 14 & 4 & $(9,0,2,0,0,3,2,0,4,0,3,0,1,0,0)$ & $\frac{1}{315}$ \\
\hline $40 a$ & & 4 & 4 & 14 & & $\{(4,10),(3,8), *\} \succ\{(7,18), *\}$ & $\frac{31^{5}}{2520}$ \\
\hline $40 b$ & & 4 & 5 & 14 & & $\{(2,5),(6,16), *\} \succ\{(8,21), *\}$ & $\frac{2520}{1260}$ \\
\hline 41 & $(0,0,0,1,1,1,0,1,1)$ & 5 & 11 & 13 & 2 & $(5,0,1,0,0,0,2,0,1,0,2,0,0,0,0)$ & $\frac{1}{252}$ \\
\hline 42 & $(0,0,0,1,1,1,0,1,1)$ & 3 & 6 & 14 & 3 & $(6,1,0,1,0,1,2,0,3,0,2,0,1,0,0)$ & $\frac{1}{770}$ \\
\hline 43 & $(0,0,0,1,1,1,0,1,1)$ & 4 & 8 & 14 & 3 & $(7,0,1,1,0,1,1,1,2,0,2,0,1,0,0)$ & $\frac{71}{27720}$ \\
\hline $43 a$ & & 2 & 4 & 14 & & $\{(4,11),(1,3), *\} \succ\{(5,14), *\}$ & $\frac{1}{2520}$ \\
\hline $43 b$ & & 3 & 4 & 14 & & $\{(2,5),(3,8), *\} \succ\{(5,13), *\}$ & $\frac{23}{36036}$ \\
\hline $43 c$ & & 3 & 5 & 14 & & $\{(7,16),(7,19), *\}$ & $\frac{31}{31920}$ \\
\hline 44 & $(0,0,0,1,1,1,0,1,1)$ & 5 & 9 & 14 & 3 & $(7,0,1,1,0,1,2,0,3,0,1,1,0,0,0)$ & $\frac{43}{13860}$ \\
\hline $44 a$ & & 4 & 4 & 14 & & $\{(2,5),(6,16), *\} \succ\{(8,21), *\}$ & $\frac{1}{1386}$ \\
\hline $44 b$ & & 3 & 3 & 14 & & $\{(7,16),(5,13), *\}$ & $\frac{1300}{16016}$ \\
\hline $44 c$ & & 4 & 6 & 14 & & $\{(7,16),(5,18), *\}$ & $\frac{1}{720}$ \\
\hline $44 d$ & & 4 & 4 & 14 & & $\{(5,13),(5,18), *\}$ & $\frac{1}{2184}$ \\
\hline 45 & $(0,0,0,1,1,1,1,0,1)$ & 4 & 7 & 14 & 2 & $(3,0,2,0,0,0,1,0,3,0,1,0,1,0,0)$ & $\frac{1}{504}$ \\
\hline 46 & $(0,0,0,1,1,1,1,1,0)$ & 4 & 7 & 14 & 3 & $(6,0,2,0,0,2,1,0,3,1,1,0,1,0,0)$ & $\frac{1}{504}$ \\
\hline $46 a$ & & 3 & 3 & 16 & & $\{(2,5),(3,8), *\} \succ\{(5,13), *\}$ & $\frac{1}{16380}$ \\
\hline $46 b$ & & 3 & 6 & 14 & & $\{(3,10),(2,7), *\} \succ\{(5,17), *\}$ & $\frac{7}{6120}$ \\
\hline 47 & $0,0,0,1,1,1,1,1,1)$ & 2 & 3 & 16 & 2 & $(3,1,0,1,0,0,1,0,2,1,0,0,1,0,0)$ & $\frac{1}{9240}$ \\
\hline 48 & $0,0,0,1,1,1,1,1,1)$ & 3 & 5 & 14 & 2 & $(4,0,1,1,0,0,0,1,1,1,0,0,1,0,0)$ & $\frac{190}{13860}$ \\
\hline $48 a$ & & 2 & 3 & 14 & & $\{(4,9),(3,7), *\} \succ\{(7,16), *\}$ & $\frac{1}{2640}$ \\
\hline 49 & $(0,0,0,1,1,1,1,1,1)$ & 4 & 7 & 14 & 3 & $(5,1,1,0,0,2,1,0,4,0,2,0,1,0,0)$ & $\frac{2470}{277^{20}}$ \\
\hline $49 a$ & & 4 & 6 & 14 & & $\{(5,11),(4,9), *\} \succ\{(9,20), *\}$ & $\frac{1}{840}$ \\
\hline 50 & $(0,0,0,1,1,1,1,1,1)$ & 5 & 9 & 14 & 3 & $(6,0,2,0,0,2,0,1,3,0,2,0,1,0,0)$ & $\frac{41}{13860}$ \\
\hline $50 a$ & & 3 & 5 & 14 & & $\{(4,11),(1,3), *\} \succ\{(5,14), *\}$ & $\frac{1}{1260}$ \\
\hline 51 & $(0,0,0,1,1,1,1,1,1)$ & 6 & 10 & 14 & 3 & $(6,0,2,0,0,2,1,0,4,0,1,1,0,0,0)$ & $\frac{1970}{27720}$ \\
\hline $51 a$ & & 5 & 4 & 14 & & $\{(4,10),(3,8), *\} \succ\{(7,18), *\}$ & $\frac{1}{1386}$ \\
\hline $51 b$ & & 5 & 5 & 14 & & $\{(5,13),(5,18), *\}$ & $\frac{1}{1170}$ \\
\hline 52 & $(0,0,1,0,0,1,0,1,0)$ & 3 & 7 & 14 & 2 & $(4,0,0,1,0,2,2,0,2,0,0,0,0,0,1)$ & $\frac{1}{420}$ \\
\hline $52 a$ & & 2 & 3 & 18 & & $\{(2,5),(3,8), *\} \succ\{(5,13), *\}$ & $\frac{1}{2184}$ \\
\hline 53 & $(0,0,1,0,0,1,1,1,0)$ & 4 & 8 & 14 & 2 & $(3,0,1,0,0,3,1,0,3,0,0,0,0,0,1)$ & $\frac{1}{360}$ \\
\hline $53 a$ & & 3 & 4 & 15 & & $\{(2,5),(3,8), *\} \succ\{(5,13), *\}$ & $\frac{1}{1170}$ \\
\hline 54 & $(0,0,1,0,1,0,0,1,0)$ & 2 & 4 & 14 & 2 & $(2,0,0,2,0,3,1,0,1,0,1,0,0,0,0)$ & $\frac{1}{840}$ \\
\hline 55 & $(0,0,1,0,1,0,0,1,0)$ & 2 & 2 & 14 & 3 & $(4,0,0,3,0,4,1,0,3,0,0,1,0,0,0)$ & $\frac{1}{3080}$ \\
\hline 56 & $(0,0,1,0,1,0,1,1,0)$ & 3 & 5 & 14 & 2 & $(1,0,1,1,0,4,0,0,2,0,1,0,0,0,0)$ & $\frac{1}{630}$ \\
\hline $56 a$ & & 2 & 3 & 14 & & $\{(4,9),(3,7), *\} \succ\{(7,16), *\}$ & $\frac{1}{1680}$ \\
\hline 57 & $(0,0,1,0,1,0,1,1,0)$ & 3 & 3 & 14 & 3 & $(3,0,1,2,0,5,0,0,4,0,0,1,0,0,0)$ & $\frac{1}{1386}$ \\
\hline 58 & $(0,0,1,0,1,1,0,1,0)$ & 3 & 6 & 14 & 3 & $(4,0,1,1,0,4,2,0,2,0,1,0,1,0,0)$ & $\frac{1}{630}$ \\
\hline $58 a$ & & 2 & 4 & 14 & & $\{(4,9),(3,7), *\} \succ\{(7,16), *\}$ & $\frac{1}{1680}$ \\
\hline 59 & $(0,0,1,0,1,1,0,1,1)$ & 2 & 4 & 14 & 2 & $(2,0,0,2,0,2,1,1,0,0,0,0,1,0,0)$ & $\frac{1}{3080}$ \\
\hline $59 a$ & & 2 & 3 & 14 & & $\{(3,8),(4,11), *\} \succ\{(7,19), *\}$ & $\frac{1}{2660}$ \\
\hline 60 & $(0,0,1,0,1,1,1,1,0)$ & 4 & 7 & 14 & 3 & $(3,0,2,0,0,5,1,0,3,0,1,0,1,0,0)$ & $\frac{1}{504}$ \\
\hline $60 a$ & & 3 & 3 & 15 & & $\{(2,5),(3,8), *\} \succ\{(5,13), *\}$ & $\frac{1}{16380}$ \\
\hline 61 & $(0,0,1,0,1,1,1,1,1)$ & 2 & 3 & 15 & 2 & $(0,1,0,1,0,3,1,0,2,0,0,0,1,0,0)$ & $\frac{1}{9240}$ \\
\hline 62 & $(0,0,1,0,1,1,1,1,1)$ & 3 & 5 & 14 & 2 & $(1,0,1,1,0,3,0,1,1,0,0,0,1,0,0)$ & $\frac{190}{13860}$ \\
\hline $62 a$ & & 2 & 3 & 14 & & $\{(4,9),(3,7), *\} \succ\{(7,16), *\}$ & $\frac{1}{2640}$ \\
\hline 63 & $(0,0,1,1,1,1,1,1,1)$ & 3 & 4 & 14 & 3 & $(5,0,1,2,0,1,1,1,3,0,1,0,0,0,1)$ & $\frac{10}{5544}$ \\
\hline
\end{tabular}


Proof. Assume $B=B_{*}$. We hope to deduce a contradiction.

(1). If $P_{14} \geq 2$, then Proposition 2.30 implies $K^{3} \geq \frac{11}{37800}>\frac{1}{3437}$. Thus $B \neq B_{4 a}, B_{9}, B_{16 c}, B_{24}, B_{27 a}, B_{29 a}, B_{44 b}, B_{63}$.

(2). If $P_{15} \geq 2$, then Proposition 2.30 implies $K^{3} \geq \frac{11}{46080}>\frac{1}{4190}$. Hence $B \neq B_{60 a}, B_{61}$.

(3). If $P_{16} \geq 2$, then Proposition 2.30 implies $K^{3} \geq \frac{11}{55488}>\frac{1}{5045}$. Hence $B \neq B_{46 a}, B_{47}$.

(4). If $P_{18} \geq 2$, then Proposition 2.30 implies $K^{3} \geq \frac{11}{77976}>\frac{1}{7089}$. Thus $B \neq B_{20 a}, B_{22}$.

(5). Besides, we see $P_{6}\left(B_{33 a}\right)=1, P_{16}\left(B_{33 a}\right)=2$ but $P_{22}\left(B_{33 a}\right)=1$, a contradiction. So $B \neq B_{33 a}$.

(6). For cases 16a, 18a, 21a, 52a, and case 55, one has $P_{17}\left(B_{*}\right)=0$. But since $P_{8}\left(B_{21 a}\right)=P_{9}\left(B_{21 a}\right)=1, B \neq\left(B_{21 a}\right)$. Also for case 52a and case 55 , since $P_{5}\left(B_{*}\right)=P_{12}\left(B_{*}\right)=1$, we see $B \neq B_{52 a}, B_{55}$. For case 18a, since $P_{6}\left(B_{18 a}\right)=P_{11}\left(B_{18 a}\right)=1$, we see $B \neq B_{18 a}$. Finally, since $P_{19}\left(B_{16 a}\right)=-1$, we see $B \neq B_{16 a}$.

q.e.d.

Theorem 4.6. Assume $\chi\left(\mathcal{O}_{X}\right)>1$. Then $K_{X}^{3} \geq \frac{1}{2660}$. Furthermore, $K_{X}^{3}=\frac{1}{2660}$ if, and only if, $P_{2}=0$ and either $\chi=3, B=B_{11 a}$, or $\chi=2$, $B_{59 a}$.

Proof. If $P_{m_{0}} \geq 2$ for some positive integer $m_{0} \leq 12$, then Proposition 2.30 implies $K_{X}^{3} \geq \frac{11}{24336}>\frac{1}{2213}>\frac{1}{2660}$.

Assume $P_{m} \leq 1$ for $m \leq 12$. Then we have seen $B \geq B_{*}$ where $B_{*}$ is one in Table $\mathrm{C}$ excluding those cases listed in Proposition 4.5.

We can see $K^{3}\left(B_{11 a}\right)=K^{3}\left(B_{59 a}\right)=\frac{1}{2660}$.

We pick out those cases with $K^{3}\left(B_{*}\right)<\frac{1}{2660}$. They are cases $4 \mathrm{a}, 16 \mathrm{a}$, 16c, 18a, 20a, 21a, 27a, 29a, 33a, 44b, 46a, and case 60a. In all these cases, Corollary 4.5 says $B \neq B_{*}$. Thus $B \succ B_{*}$. In order to prove the theorem, we need to study the one-step unpacking of $B_{*}$ case by case.

First we consider case $4 \mathrm{a}$ and case 29a. It's obtained by 2-steps of packing from $B_{4}$ :

$B_{4}=\{(2,6),(4,11), *\} \succ B_{4.5}:=\{(1,3),(5,14), *\} \succ\{(6,17), *\}=B_{4 a}$.

By $\left[\mathbf{4}\right.$, Lemma 3.6], we get $K_{X}^{3}=K^{3}(B) \geq K^{3}\left(B_{4.5}\right)=\frac{1}{630}>\frac{1}{2660}$. Similarly, we also get $K_{X}^{3}>\frac{1}{2660}$ for case 29 a.

Next, we consider cases 18a, 20a, 21a, 46a, 52a, 60a. The common property is that they are obtained by a 1-step packing from $B^{(12)}$. So the only possibility is $B^{(12)}=B$. Thus $K_{X}^{3}=K^{3}\left(B_{18}\right)$ or $K^{3}\left(B_{20}\right)$ or $K^{3}\left(B_{21}\right)$ or $K^{3}\left(B_{46}\right)$ or $K^{3}\left(B_{52}\right)$ or $K^{3}\left(B_{60}\right)$. In a word, $K_{X}^{3}>\frac{1}{2660}$.

The remaining cases are $16 \mathrm{a}, 16 \mathrm{c}, 27 \mathrm{a}$, and $44 \mathrm{~b}$. For case $44 \mathrm{~b}$, there are two intermediate baskets dominating $B_{44 c}$ or $B_{44 d}$, respectively. Thus, in particular, $K_{X}^{3}>\frac{1}{2184}$. For case $27 \mathrm{a}$, it's obtained from $B_{27}$ by 
3-steps of packing, namely,

$$
\begin{aligned}
& B_{27}=\{3 \times(2,5),(5,8), *\} \succ\{2 \times(2,5),(5,13), *\} \\
\succ & B_{27.5}:=\{(2,5),(7,18), *\} \succ\{(9,23), *\}=B_{27 a} .
\end{aligned}
$$

Thus we see $B \succcurlyeq B_{27.5}$ and $K_{X}^{3} \geq K^{3}\left(B_{27.5}\right)=\frac{1}{1386}>\frac{1}{2660}$. Finally, we consider cases $16 \mathrm{a}$ and $16 \mathrm{c}$. We know

$$
B_{16}=\{(4,9),(3,7),(2,5),(2,5),(3,8),(3,8), *\} .
$$

The 1-step packing of $B_{16}$ yields

$$
B_{16.5}:=\{(4,9),(3,7),(2,5),(5,13),(3,8), *\},
$$

and the 1-step prime packing of $B_{16.5}$ is either $B_{16 a}$ or $B_{16 c}$. Thus, if $B \succcurlyeq$ $B_{16.5}$, then $K_{X}^{3} \geq K^{3}\left(B_{16.5}\right)=\frac{85}{72072}>\frac{1}{848}$. The other intermediate basket dominating $B_{16 a}$ and $B_{16 c}$ is

$$
B_{16.6}:=\{(7,16),(2,5),(2,5),(3,8),(3,8), *\}
$$

with $K^{3}\left(B_{16.6}\right)=\frac{13}{6160}>\frac{1}{474}$. There are no other ways to obtain either $B_{16 a}$ or $B_{16 c}$ beginning from $B^{(16)}$. The theorem is proved. q.e.d.

Corollary 4.7. Assume $\chi\left(\mathcal{O}_{X}\right)>1$. Then $P_{24} \geq 2$.

Proof. By [4, Theorem 4.15], we know either $P_{10} \geq 2$ or $P_{24} \geq 2$. When $q(X)>0$, the statement follows from [3]. So we may assume $q(X)=0$.

If $P_{10} \geq 2$, we take $m_{0}=10$ and study $\varphi_{10}$. Keep the same notation as in 2.3. By Lemma 2.32, $f$ must be of type $I I I, I I, I_{p}$. Proposition 2.15 (i), Theorem 2.20 (1), and Theorem 2.22 (1) imply $P_{24} \geq 2$. q.e.d.

Theorem 4.8. Assume $\chi\left(\mathcal{O}_{X}\right)>1$. Then $P_{m_{0}} \geq 2$ for some positive integer $m_{0} \leq 18$. In particular, $\mu_{1} \leq 18$.

Proof. Assume $P_{m} \leq 1$ for all $m \leq 12$. Then Table $\mathrm{C}$ tells us that

$$
B^{(12)} \succcurlyeq B \succcurlyeq B_{\min }
$$

where $B_{\min }$ is of certain type in Table C. Since, in Table C, we have seen $\mu_{1}\left(B_{\text {min }}\right) \leq 18$, thus $\left[4\right.$, Lemma 3.6] implies $\mu_{1}(X) \leq \mu_{1}\left(B_{\text {min }}\right) \leq 18$. q.e.d.

Theorem 4.9. Assume $\chi\left(\mathcal{O}_{X}\right)>1$. Then $\rho_{0}(X) \leq 27$.

Proof. The statement follows from $[\mathbf{3}]$ when $q(X)>0$. Assume $q(X)=0$ from now on.

If $P_{m_{0}} \geq 2$ for some $m_{0} \leq 12$, then the induced fibration $f$ from $\varphi_{m_{0}}$ is of type $I I I, I I$, or $I_{p}$ by Lemma 2.32. Thus Proposition 2.15(i), Theorem 2.20(1), and Theorem 2.22(1) imply that $P_{m}>0$ for all $m \geq$ 27.

If $P_{m} \leq 1$ for all $m \leq 12$, we have a complete classification (cf. Table C). For each $B_{\min }$ in Table C, we observed that $P_{m}>0$ for all $47 \geq m \geq 24$. This is enough to assert $P_{m}>0$ for all $m \geq 24$. We are done. 


\section{Pluricanonical birationality}

In this section, we mainly study the birationality of $\varphi_{m}$. Then we can conclude our main theorems. Let $X$ be a projective minimal 3 -fold of general type. First, we recall several known theorems.

Theorem $5.1([\mathbf{3}])$. Assume $q(X):=h^{1}\left(\mathcal{O}_{X}\right)>0$. Then $\varphi_{m}$ is birational for all $m \geq 7$.

Theorem 5.2. ([7], [Theorem 0.1]) Assume $P_{m_{0}} \geq 2$ for some positive integer $m_{0}$. Then $\varphi_{m}$ is birational onto its image for all $m \geq$ $5 m_{0}+6$.

Theorem $5.3([\mathbf{8}])$. Assume $\chi\left(\mathcal{O}_{X}\right) \leq 0$. Then $\varphi_{m}$ is birational for all $m \geq 14$.

We need the following lemma to prove our main theorems.

Lemma 5.4. Assume $P_{m_{0}}(X) \geq 2$ for some positive integer $m_{0}$. Keep the same notation as in 2.3 and assume $f$ is of type $I_{p}$ or $I_{n}$. Suppose $|G|$ is a base point free linear system on $S$. If there exists an integer $m_{1}>0$ with $\left.m_{1} \pi^{*}\left(K_{X}\right)\right|_{S} \geq G$, then Assumption 2.9(2) is satisfied for all integers

$$
m \geq \max \left\{\rho_{0}+m_{0}+m_{1}, m_{0}+m_{1}+2\right\} .
$$

Proof. Since

$$
\begin{aligned}
& K_{S}+\left\lceil(m-1) \pi^{*}\left(K_{X}\right)-S-\frac{1}{p} E_{m_{0}}^{\prime}\right]_{\mid S} \\
\geq & K_{S}+(m-1) \pi^{*}\left(K_{X}\right)_{\mid S}-\left(S+E_{m_{0}}^{\prime}\right)_{\mid S} \\
\geq & \left(m-m_{0}\right) \pi^{*}\left(K_{X}\right)_{\mid S} \geq\left(m-m_{0}-m_{1}\right) \pi^{*}\left(K_{X}\right)_{\mid S}+G
\end{aligned}
$$

and

$$
\begin{aligned}
& K_{S}+\left(m-m_{0}-1\right) \pi^{*}\left(K_{X}\right)_{\mid S} \\
\geq & K_{S}+\left(m-m_{0}-m_{1}-1\right) \pi^{*}\left(K_{X}\right)_{\mid S}+G,
\end{aligned}
$$

Lemma 2.17 implies that $\left|K_{S}+\left\lceil(m-1) \pi^{*}\left(K_{X}\right)-S-\frac{1}{p} E_{m_{0}}^{\prime}\right\rceil_{\mid S}\right|$ can distinguish different generic irreducible elements of $|G|$ when $m \geq \rho_{0}+$ $m_{0}+m_{1}$ and $m \geq m_{0}+m_{1}+2$.

q.e.d.

Theorem 5.5. Let $X$ be a projective minimal 3-fold of general type with $\chi\left(\mathcal{O}_{X}\right)=1$. Then $\varphi_{m}$ is birational for all $m \geq 40$.

Proof. If $P_{m_{0}} \geq 2$ for some $m_{0} \leq 6$, then, by Theorem 5.2, $\varphi_{m}$ is birational for $m \geq 36$.

Assume $P_{m} \leq 1$ for all $m \leq 6$. Then, by Corollary $3.12, \mathscr{B}(X)$ either dominates a minimal basket in

$$
\left\{B_{2,1}, B_{2,2}, B_{3,1} \sim B_{3,5}, B_{5,1} \sim B_{5,3}, B_{6,1} \sim B_{6,6}, B_{8,1}, B_{8,2}\right\}
$$

or dominates the basket $B_{210}$. We have known $P_{m}(X) \geq P_{m}\left(B_{*, *}\right)$. By analyzing all the above baskets, we see a common property that there 
is a pair of positive integers $\left(n_{0}, n_{1}\right)$ satisfying $P_{n_{0}} \geq 2, P_{n_{1}} \geq 3$, and one of the following conditions:

(1) $n_{0} \leq 10, n_{1} \leq 12$ (see cases III-2, III-3, III-4, VI-6);

(2) $n_{0} \leq 9, n_{1} \leq 13$ (for the remaining cases).

By Theorem 3.14 (1), we know $\rho_{0} \leq 7$. We set $m_{0}=n_{1}$. Keep the same notation as in 2.3. Our proof is organized according to the type of $f$. Note that $P_{m_{0}} \geq 3$ and $m_{0} \leq 13$. By Theorem 5.1, we only need to care about the situation $q(X)=0$.

Case 1. $f$ is of type $I_{3}$.

Take $G$ to be the movable part of $\left|2 \sigma^{*}\left(K_{S_{0}}\right)\right|$. Claim A implies that Assumption 2.9(2) is satisfied whenever $m \geq 35 \geq \rho_{0}+2 m_{0}+2$. Clearly, by Lemma 2.16, Assumption 2.9(1) is also satisfied. As seen in the later part of 2.28 , we can take a rational number $\beta \mapsto \frac{p}{2 m_{0}+2 p} \geq \frac{1}{m_{0}+2}$. Now inequality (2.2) gives $\xi \geq \frac{4}{45}$. Take $m=35$. Then $\alpha=\left(35-1-\frac{m_{0}}{2}-\right.$ $\left.\frac{1}{\beta}\right) \xi \geq \frac{10}{9}>1$. Theorem 2.11 gives $\xi \geq \frac{4}{35}$. Take $m=32$. Then $\alpha>1$. We will see $\xi \geq \frac{1}{8}$ similarly. Now, for $m \geq 39, \alpha \geq\left(39-1-\frac{13}{2}-15\right) \xi \geq$ $\frac{33}{16}>2$. Theorem 2.11 says that $\varphi_{m}$ is birational for all $m \geq 39$.

Case 2. $f$ is of type $I I$ or $I I I$.

We take $\tilde{m}_{0}=n_{0}$ and $m_{1}=n_{1}$. We still use the mechanics of 2.3 to study $\varphi_{\tilde{m}_{0}}$ instead of $\varphi_{m_{0}}$. But most notations will use the symbol $\sim$. Note that $\tilde{m}_{0} \leq 10$ and $P_{\tilde{m}_{0}} \geq 2$.

If $\tilde{f}$ is of type $I I$ or $I I I$, Theorem 2.20(3) and Theorem 2.22(3) imply that $\varphi_{m}$ is birational for $m \geq 38$.

If $\tilde{f}$ is of type $I_{n}$ or $I_{p}$, we take $\tilde{G}$ to be the movable part of $\left|M_{m_{1}}\right|_{\tilde{S}} \mid$, where $\tilde{S}$ is a generic irreducible element of $\left|M_{\tilde{m}_{0}}\right|$. Clearly, $h^{0}\left(\tilde{S},\left.M_{m_{1}}\right|_{\tilde{S}}\right)$ $\geq 2$ since $\operatorname{dim} \varphi_{m_{1}}(X) \geq 2$. Thus we are in the situation with $\left.m_{1} \pi^{*}\left(K_{X}\right)\right|_{\tilde{S}} \geq \tilde{G}$. We may always take a sufficiently good $\tilde{\pi}$ instead of $\pi$. Now Lemma 2.16 and Lemma 5.4 imply that Assumptions 2.9(1) and 2.9(2) are simultaneously satisfied for $m \geq 30 \geq \rho_{0}+\tilde{m}_{0}+m_{1}$. Finally, we study the value of $\alpha$. Clearly, one may take $\tilde{\beta}=\frac{1}{m_{1}}$. Thus inequality (2.2) says $\xi \geq \frac{2}{1+\tilde{m_{0}}+m_{1}}$. For situations (1) and (2), we have $\xi \geq \frac{2}{23}$. Take $m=35$. Then $\alpha \geq \frac{24}{23}>1$. Theorem 2.11 gives $\xi \geq \frac{4}{35}$. Take $m=32$. Then, similarly, we get $\xi \geq \frac{1}{8}$. Take $m \geq 40$. Then $\alpha \geq \frac{17}{8}>2$. Theorem 2.11 implies that $\varphi_{m}$ is birational for all $m \geq 40$. We are done.

q.e.d.

L. Zhu [30] showed $\varphi_{m}$ is birational for $m \geq 46$.

Theorem 5.6. Let $X$ be a projective minimal 3-fold of general type with $\chi\left(\mathcal{O}_{X}\right)>1$. Then $\varphi_{m}$ is birational for all $m \geq 73$.

Proof. By Theorem 5.1, we only need to consider the situation $q(X)=$ 0 . According to Lemma 2.32, the induced fibration $f$ from $\varphi_{m_{0}}$ is of type $I I I, I I$, or $I_{p}$. 
If $P_{m_{0}} \geq 2$ for some $m_{0} \leq 16$, then, by Theorems $2.20,2.22$, and 2.25 , $\varphi_{m}$ is birational for all $m \geq 69$. Assume $P_{m} \leq 1$ for all $m \leq 16$. Then we have a complete classification for $B_{\min }$ as in Table C. More precisely, we see $B \succcurlyeq B_{2 a}, B \succcurlyeq B_{3 a}$ and $B \succ B_{20 a}, B \succ B_{52 a}$, noting that case No.22 doesn't happen by Proposition 4.5. As we have observed in the proof of Theorem 4.6, for cases No. 20a and No. 52a, we actually have $B=B_{20}$ and $B=B_{52}$. Thus we see $P_{14}(X) \geq 2$ in both cases, a contradiction. We are left to study cases No. $2 \mathrm{a}$ and No. 3a, which correspond to two formal baskets, $\left(B_{2 a}, 2,0\right)$ and $\left(B_{3 a}, 3,0\right)$, where

$$
\begin{gathered}
B_{2 a}=\{4 \times(1,2),(4,9),(2,5),(5,13), 3 \times(1,3), 2 \times(1,4)\}, \\
B_{3 a}=\{6 \times(1,2),(5,11), 3 \times(2,5),(5,13), 4 \times(1,3),(2,7), 2 \times(1,4)\} .
\end{gathered}
$$

The computation gives the following datum:

\begin{tabular}{c|cccc}
\hline & $\rho_{0}$ & $\mu_{1}$ & $\mu_{2}$ & $\mu_{3}$ \\
\hline$B_{2 a}$ & 20 & 18 & 24 & 30 \\
$B_{3 a}$ & 20 & 18 & 20 & 30 \\
\hline
\end{tabular}

When $B \succcurlyeq B_{3 a}$, we have $P_{20}(X)=P_{20}(B) \geq P_{20}\left(B_{3 a}\right) \geq 3$. Theorems 2.20 and 2.29 imply that $\varphi_{m}$ is birational for all $m \geq 66$ unless $f$ is type $I I$. Indeed, if $f$ is of type $I I$ and $m_{0}=20$, at least we have $\xi \geq \frac{2}{31}$, following the argument in 2.21. Take $m=57$; we have $\alpha>1$ and hence $\xi \geq \frac{4}{57}$. Now take $m \geq 70$; we have $\alpha=(70-41) \frac{4}{57}>2$. Thus $\varphi_{m}$ is birational for all $m \geq 70$.

Now the theorem follows from the following claim.

Claim B. When $B \succcurlyeq B_{2 a}, \varphi_{m}$ is birational for all $m \geq 73$.

The proof is similar to that of Theorem 5.5, Case 1 and Case 2. We have known $\rho_{0} \leq 20$. We can find two numbers $n_{0} \leq 18$ and $n_{1} \leq 24$ with $P_{n_{0}}(X) \geq 2$ and $P_{n_{1}}(X) \geq 3$. First, we set $m_{0}=n_{1}$. Keep the same notation as in 2.3. Our proof is organized according to the type of $f$. Note that $P_{m_{0}} \geq 3$ and $m_{0} \leq 24$.

Case i. $f$ is of type $I_{3}$.

By Lemma 2.32, $f$ must be of type $I_{p}$. Take $G=2 \sigma^{*}\left(K_{S_{0}}\right)$. Claim A implies that Assumption 2.9(2) is satisfied whenever $m \geq 70 \geq$ $\rho_{0}+2 m_{0}+2$. Clearly, by Lemma 2.16, Assumption 2.9(1) is also satisfied. As seen in the latter part of 2.28 , we can take a rational number $\beta \mapsto \frac{p}{2 m_{0}+2 p} \geq \frac{1}{m_{0}+2}$. Note that $|G|$ is base point free, and we have $\operatorname{deg}\left(K_{C}\right) \geq 6$. Now inequality (2.2) gives $\xi \geq \frac{2}{13}$. For $m \geq 70$, $\alpha \geq(70-1-12-26) \xi>2$. Theorem 2.11 says that $\varphi_{m}$ is birational for all $m \geq 70$.

Case ii. $f$ is of type $I I$ or $I I I$.

We take $\tilde{m}_{0}=n_{0}$ and $m_{1}=n_{1}$. We still use the mechanics of 2.3 to study $\varphi_{\tilde{m}_{0}}$ instead of $\varphi_{m_{0}}$. Noting that $\tilde{m}_{0} \leq 18$, when $\tilde{f}$ is of type $I I I$ 
or $I I$, Theorems 2.20 and 2.22 imply that $\varphi_{m}$ is birational for all $m \geq 66$. We are left to study the situation with $\tilde{f}$ being of type $I$. We take $\tilde{G}$ to be the movable part of $\left|M_{m_{1}}\right|_{\tilde{S}} \mid$. Clearly, $h^{0}\left(\tilde{S},\left.M_{m_{1}}\right|_{\tilde{S}}\right) \geq 2$ since $\operatorname{dim} \varphi_{m_{1}}(X) \geq 2$. Thus we are in the situation with $\left.m_{1} \pi^{*}\left(K_{X}\right)\right|_{\tilde{S}} \geq \tilde{G}$. Now Lemma 2.16 and Lemma 5.4 imply that Assumptions 2.9(1) and $2.9(2)$ are simultaneously satisfied for $m \geq 62 \geq \rho_{0}+\tilde{m}_{0}+m_{1}$. Clearly, one may take $\tilde{\beta}=\frac{1}{m_{1}}$. Thus inequality (2.2) says $\xi \geq \frac{2}{1+m_{0}+m_{1}} \geq \frac{2}{43}$.

Take $m=65$. Then $\alpha \geq \frac{44}{43}>1$. Theorem 2.11 gives $\xi \geq \frac{4}{65}$. Take $m=60$. Then similarly we get $\xi \geq \frac{1}{15}$. Take $m=59$. Then we shall get $\xi \geq \frac{4}{59}$. Take $m=58$ and we obtain $\xi \geq \frac{2}{29}$. Eventually, for $m \geq 73$, we see $\alpha>2$ and Theorem 2.11 implies that $\varphi_{m}$ is birational for all $m \geq 73$. We are done.

q.e.d.

We have proved all the main results. Indeed, Theorem 1.1 follows from Theorem 5.1, Theorem 5.3, Theorem 5.5, and Theorem 5.6. Theorem 1.2 follows from Theorem 3.11 and Theorem 4.6.

Finally, we would like to ask the following:

Question 5.7. Can one find an optimal lower bound for $K^{3}$ ?

The following problem is very interesting.

Open Problem 5.8. Can one find a minimal 3-fold $X$ of general type with $q(X)=0$ and $\chi\left(\mathcal{O}_{X}\right)>1$ ?

\section{References}

[1] W. Barth, C. Peters \& A. Van de Ven, Compact complex surfaces, Ergebnisse der Mathematik und ihrer Grenzgebiete (3) 4, Springer-Verlag, Berlin, 1984. x+304 pp., MR 0749574, Zbl 1036.14016.

[2] E. Bombieri, Canonical models of surfaces of general type, Inst. Hautes Etudes Sci. Publ. Math., 42 (1973), 171-219, MR 0318163, Zbl 0259.14005.

[3] J. A. Chen \& C. D. Hacon, Pluricanonical systems on irregular 3-folds of general type, Math. Z., 255 (2007), no. 2, 343-355, MR 2262735, Zbl 1094.14502.

[4] J. A. Chen \& M. Chen, Explicit birational geometry of 3-folds of general type, I, Ann. Sci. École Norm. Sup., 43 (2010), 365-394.

[5] J. A. Chen \& M. Chen, The Canonical volume of 3-folds of general type with $\chi \leq$ 0, J. London Math. Soc., 78 (2008), 693-706, MR 2456899, Zbl 1156.14009.

[6] M. Chen, Canonical stability in terms of singularity index for algebraic threefolds, Math. Proc. Camb. Phil. Soc., 131 (2001), 241-264, MR 1857118, Zbl 1068.14045 .

[7] M. Chen, On the Q-divisor method and its application, J. Pure Appl. Algebra, 191 (2004), 143-156, MR 2048311, Zbl 1049.14034.

[8] M. Chen \& K. Zuo, Complex projective threefolds with non-negative canonical Euler-Poincare characteristic, Comm. Anal. Geom., 16 (2008), 159-182, MR 2411471, Zbl 1149.14034.

[9] C. Ciliberto, The bicanonical map for surfaces of general type, Proc. Symposia in Pure Math., 62 (1997), 57-83, MR 1492518, Zbl 0929.14022. 
[10] C. D. Hacon \& J. $\mathrm{M}^{\mathrm{c}}$ Kernan, Boundedness of pluricanonical maps of varieties of general type, Invent. Math., 166 (2006), 1-25, MR 2242631, Zbl 1121.14011.

[11] A. R. Iano-Fletcher. Contributions to Riemann-Roch on Projective 3-folds with Only Canonical Singularities and Applications, Proceedings of Symposia in Pure Mathematics, 46 (1987), 221-231, MR 0927958, Zbl 0662.14026.

[12] A. R. Iano-Fletcher, Working with weighted complete intersections, In Explicit birational geometry of 3-folds, London Mathematical Society, Lecture Note Series, 281, Cambridge University Press, Cambridge, 2000, MR 1798982.

[13] Y. Kawamata, A generalization of Kodaira-Ramanujam's vanishing theorem, Math. Ann., 261 (1982), 43-46, MR 0675204, Zbl 0476.14007.

[14] Y. Kawamata, K. Matsuda \& K. Matsuki, Introduction to the minimal model problem, Adv. Stud. Pure Math., 10 (1987), 283-360, MR 0946243, Zbl 0672.14006

[15] J. Kollár, Higher direct images of dualizing sheaves I, Ann. Math., 123(1986), 11-42, MR 0825838, Zbl 0598.14015; II, ibid. 124 (1986), 171-202, MR 0847955, Zbl 0605.14014.

[16] J. Kollár \& S. Mori, Birational geometry of algebraic varieties, 1998, Cambridge Univ. Press, MR 1658959, Zbl 0926.14003.

[17] M. Mendes Lopes, Adjoint systems on surfaces, Boll. Unione Mat. Ital., (7)10-A (1996), 169-179, MR 1386254, Zbl 0861.14023.

[18] Y. Miyaoka, The Chern classes and Kodaira dimension of a minimal variety, Algebraic Geometry, Sendai, 1985. Adv. Stud. Pure Math., 10 (1987), 449-476, MR 0946247, Zbl 0648.14006.

[19] M. Reid, Canonical 3-folds, in A. Beauville, editor, Géométric Algébrique Angers, pages 273-310, Sijthoff \& Noordhoff, 1980, MR 0605348, Zbl 0451.14014.

[20] M. Reid, Minimal models of canonical 3-folds, Adv. Stud. Pure Math., 1 (1983), 131-180, MR 0715649, Zbl 0558.14028.

[21] M. Reid, Young person's guide to canonical singularities, Proc. Symposia in pure Math., 46 (1987), 345-414, MR 0927963, Zbl 0634.14003.

[22] D.-K. Shin, On a computation of plurigenera of a canonical threefold, J. Alg., 309 (2007), 559-568, MR 2303193, Zbl 1116.14032.

[23] S. Takayama, Pluricanonical systems on algebraic varieties of general type, Invent. Math., 165 (2006), 551-587, MR 2242627, Zbl 1108.14031.

[24] S. G. Tankeev, On n-dimensional canonically polarized varieties and varieties of fundamental type, Izv. A. N. SSSR, Ser. Math., 35 (1971), 31-44, MR 0277528, Zbl 0248.14005.

[25] H. Tsuji, Pluricanonical systems of projective varieties of general type, I, Osaka J. Math., 43 (2006), no. 4, 967-995, MR 2303558, Zbl 1142.14012.

[26] E. Viehweg, Vanishing theorems, J. reine angew. Math., 335 (1982), 1-8, MR 0667459, Zbl 0485.32019.

[27] E. Viehweg, Weak positivity and the additivity of the Kodaira dimension for certain fibre spaces, Proc. Algebraic Varieties and Analytic Varieties, Tokyo 1981. Adv. Studies in Math., 1, Kinokunya-North-Holland Publ., 1983, 329353, MR 0715656, Zbl 0513.14019.

[28] G. Xiao, Finitude de l'application bicanonique des surfaces de type général, Bull. Soc. Math. France, 113 (1985), 23-51, MR 0807825, Zbl 0611.14031. 
[29] L. Zhu, The sharp lower bound for the volume of threefolds of general type with $\chi\left(\mathcal{O}_{X}\right)=1$, Math. Zeit., 261 (2009), no. 1, 123-141, MR 2452640, Zbl 1154.14033 .

[30] L. Zhu, On birational geometry of algebraic 3-folds of general type, PhD thesis, Fudan University, 2008.

Department of Mathematics

NATIONAL TAIWAN UNIVERSITY

TAIPEI, 106

TAIWAN

E-mail address: jkchen@math.ntu.edu.tw

School of Mathematical Sciences \& LMNS

FUDAN UNIVERSITY

Shanghai, 200433

People's Republic of China

E-mail address: mchen@fudan.edu.cn 